1

\title{
Tom70-based transcriptional regulation of mitochondrial biogenesis and aging
}

\author{
Qingqing Liu ${ }^{1}$, Catherine E. Chang ${ }^{1}$, Alexandra C. Wooldredge ${ }^{1}$, Benjamin Fong ${ }^{1}$, Brian K.
} Kennedy ${ }^{1,3,4,5}$, and Chuankai Zhou ${ }^{1,2, *}$

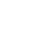

${ }^{1}$ Buck Institute for Research on Aging, 8001 Redwood Blvd, Novato, CA 94945, USA

${ }^{2}$ USC Leonard Davis School of Gerontology, University of Southern California, 3715

McClintock Ave., Los Angeles, CA 90191, USA

${ }^{3}$ Healthy Longevity Programme, Yong Loo Lin School of Medicine, National University of Singapore, 10 Medical Dr., Singapore 117597, Singapore;

${ }^{4}$ Centre for Healthy Longevity, National University Health System, Singapore, 1E Kent Ridge Rd., Singapore 119228, Singapore;

${ }^{5}$ Singapore Institute of Clinical Sciences, A(*)STAR, Singapore 117609, Singapore. 4

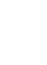

*Correspondence: KZhou@buckinstitute.org

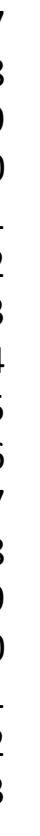




\section{Abstract}

35 Mitochondrial biogenesis has two major steps: the transcriptional activation of nuclear genome-

36 encoded mitochondrial proteins and the import of nascent mitochondrial proteins that are

37 synthesized in the cytosol. These nascent mitochondrial proteins are aggregation-prone and can

38 cause cytosolic proteostasis stress. The transcription factor-dependent transcriptional regulations

39 and the TOM-TIM complex-dependent import of nascent mitochondrial proteins have been

40 extensively studied. Yet, little is known regarding how these two steps of mitochondrial

41 biogenesis coordinate with each other to avoid the cytosolic accumulation of these aggregation-

42 prone nascent mitochondrial proteins. Here we show that in budding yeast, Tom70, a conserved

43 receptor of the TOM complex, moonlights to regulate the transcriptional activity of

44 mitochondrial proteins. Tom70's transcription regulatory role is conserved in Drosophila. The

45 dual roles of Tom70 in both transcription/biogenesis and import of mitochondrial proteins allow

46 the cells to accomplish mitochondrial biogenesis without compromising cytosolic proteostasis.

47 The age-related reduction of Tom70, caused by reduced biogenesis and increased degradation of

48 Tom70, is associated with the loss of mitochondrial membrane potential, mtDNA, and

49 mitochondrial proteins. While loss of Tom70 accelerates aging and age-related mitochondrial

50 defects, overexpressing TOM70 delays these mitochondrial dysfunctions and extends the

51 replicative lifespan. Our results reveal unexpected roles of Tom70 in mitochondrial biogenesis

52 and aging.

54 Keywords: Mitochondria; mitochondrial biogenesis; Tom70; proteostasis; aging; mitochondria

55 defect, transcriptional regulation, caloric restriction.

\section{Introduction}

58 Mitochondria play critical roles in a growing list of cellular activities, such as signaling,

59 metabolism, and inflammation. A decline in mitochondrial function, both in quality and quantity,

60 has been associated with normal aging and many age-related diseases (Gureev et al., 2019; Sun

61 et al., 2016). Mitochondrial quality and quantity are determined by mitochondrial biogenesis and

62 quality control mechanisms (Baker et al., 2011; Pickles et al., 2018; Shpilka and Haynes, 2018).

63 More than $99 \%$ of mitochondrial proteins are encoded by the nuclear genome (Fox, 2012;

64 Mootha et al., 2003; Morgenstern et al., 2017; Pagliarini et al., 2008; Vögtle et al., 2017). The 
expression of mitochondrial proteins is mainly regulated at the transcriptional level by

66

67

68

69

70

71

72

73

74

75

76

77

78

79

80

81

82

83

84

85

86

87

88

89

90

91

92

93

94

95 transcription factors that integrate cellular and environmental signals (de Winde and Grivell, 1993; Jornayvaz and Shulman, 2010; Scarpulla, 2008). For example, in budding yeast, cells sense nutrients and regulate the expression of mitochondrial proteins through Hap4, a wellstudied transcription activator of mitochondrial biogenesis (Forsburg and Guarente, 1989; McNabb et al., 1995; Olesen et al., 1987; Pinkham and Guarente, 1985; Santangelo, 2006; Schüller, 2003). In addition to transcription activation, repressors such as Mig1 and Rox1 also play a critical role to set the balance and maintain optimal expression of mitochondrial proteins in response to different growth conditions (Lowry and Zitomer, 1984; Santangelo, 2006;

Schüller, 2003; Ter Linde and Steensma, 2002; Trueblood et al., 1988).

After completing transcription, these mRNAs encoding mitochondrial proteins are translated in the cytosol and imported by the TOM-TIM complex (Wiedemann and Pfanner, 2017). Most mitochondrial proteins are selectively recognized and imported via Tom20 and Tom70 pathways, two major receptors of the TOM complex. The Tom 20 pathway is known to import nascent mitochondrial proteins that contain an $\mathrm{N}$-terminal signal peptide. A significant number of nascent mitochondrial proteins lack the typical N-terminal peptides and, instead, present the internal mitochondrial targeting signal (iMTS) that span the entire primary sequence and are imported via the Tom70 pathway (Backes et al., 2018; Sirrenberg et al., 1996). Another unique feature of the Tom70 pathway is that cytosolic chaperones, such as Hsp70, are involved in maintaining these mitochondrial proteins in unfolded states before being transferred to Tom70 for import (Hoseini et al., 2016; Young et al., 2003; Zanphorlin et al., 2016). Therefore, mitochondrial import, especially the Tom70-mediated pathway, is integrated into the cytosolic proteostasis. Indeed, the accumulation of nascent mitochondrial proteins in the cytosol triggers cytosolic stress responses, known as UPRam and mPOS (Wang and Chen, 2015; Wrobel et al., 2015). Given the aggregation-prone nature of these nascent mitochondrial proteins (Nowicka et al., 2021), a balance between the production and import of mitochondrial proteins is required to avoid the accumulation of these proteins in the cytosol.

4 Although the transcriptional regulations of mitochondrial proteins and the molecular mechanisms of mitochondrial import have been extensively studied in the past, they were investigated 
separately, and little is known regarding how these two steps of mitochondrial biogenesis coordinate with each other to avoid the cytosolic accumulation of these aggregation-prone nascent mitochondrial proteins. In this study, we use budding yeast as the model to provide evidence that the mitochondrial import receptor Tom70 moonlights to regulate the transcriptional activity of mitochondrial proteins. This transcription regulatory role of Tom70 is conserved in Drosophila melanogaster (fruit fly). Our results suggest that cells use the same molecule, Tom70, to regulate both the transcription/biogenesis and import of mitochondrial proteins so the nascent mitochondrial proteins do not compromise cytosolic proteostasis and cause cytosolic protein aggregation. Importantly, the Tom70 abundance is decreased during aging, which is associated with age-dependent loss of mitochondrial membrane potential, mtDNA, and mitochondrial proteins. While loss of Tom70 accelerates aging and age-related mitochondrial defects, overexpressing TOM70 delays these mitochondrial dysfunctions and extends the replicative lifespan. Therefore, the reduction of Tom70 is a key event contributing to the mitochondrial biogenesis and defects during aging.

\section{Results}

\section{Tom70 regulates the transcriptional activity of mitochondrial proteins.}

113 As the abundance of the TOM complex on the mitochondrial outer membrane determines how

114 fast nascent mitochondrial proteins can be imported, we started out by examining if TOM

115 proteins may also regulate the biogenesis of mitochondrial proteins so the biogenesis and import 116 of nascent mitochondrial proteins can be coordinated with each other. We used the pGAL

117 promoter to overexpress (OE) different TOM proteins and Hap4, which is known to drive 118 mitochondrial biogenesis (Lin et al., 2002). To examine their effects on mitochondrial 119 biogenesis, we randomly selected four mitochondrial proteins to represent different sub120 compartments of the organelle. The expression levels of representative mitochondrial proteins 121 were visualized by endogenous C-terminal GFP tagging and expressed from their native 122 promoters using the strains from the yeast GFP library (Huh et al., 2003). This library has been 123 extensively characterized against different proteomic results to show high accuracy and fidelity 124 (Ho et al., 2018; Newman et al., 2006). To quantitatively compare the GFP signals from different 125 strains, we imaged the cells with an avalanche photodiode (APD), which is a single-photon 126 sensitive detector (Bruschini et al., 2019). As a single GFP protein emits 21 photons in our 
127 imaging setting (Kubitscheck et al., 2000), this single-photon sensitive detector allowed us to 128 accurately compare the abundance of these mitochondrial proteins across different conditions in 129 live cells.

131 Compared to the wild type cells cultured in the same medium, we found that HAP4 OE indeed 132 could increase the expression of two out of these four randomly chosen mitochondrial proteins 133 (Figure 1A). This is consistent with previous results that HAP4 OE increases the biogenesis of 134 roughly $1 / 4$ of the mitochondrial proteome (255 out of $>1,000$ mitochondrial proteins) (Lin et al., 2002). Among these TOM proteins, only TOM70 OE increased the abundance of these 136 mitochondrial proteins (Figure 1A). Compared to HAP4 OE, TOM70 OE has a similar but 137 broader effect on these mitochondrial proteins (Figure 1A). Interestingly, Tom71, the paralog of 138 Tom70, failed to induce the expression of any of these mitochondrial proteins (Figure 1A). The effect of TOM70 OE on these mitochondrial proteins is not caused by or unique to galactose: (1)

140 all strains, including wild type and HAP4 OE, were cultured in the same galactose medium while 141 OE of Tom70, but not other TOM proteins, increased mitochondrial biogenesis; (2) similar 142 results were observed when Tom70 was overexpressed from an artificial promoter Z3EV, which

143 is inducible by the $\beta$-estradiol, in the glucose medium (Figure 1-figure supplement 1A) (McIsaac 144 et al., 2013). We then expanded the list to cover more mitochondrial proteins from all four sub145 compartments of the organelle and found TOM70 OE generally increased their abundance 146 (Figure 1B).

148 The enhanced mitochondrial biogenesis in response to TOM70 OE was also observed at the 149 transcriptional level: TOM70 OE generally increased the mRNA levels of nucleus-encoded 150 mitochondrial proteins, including the tail-anchored small TOM proteins that are known to be 151 post-translationally inserted into the mitochondrial outer membrane independently of Tom 20 and 152 Tom70 (Figure 1C) (Drwesh and Rapaport, 2020). This indicates that the transcriptional 153 activation of mitochondrial biogenesis upon TOM70 OE is not restricted to Tom70's substrates. 154 Consistent with its role in transcription activation, when Tom70 was selectively removed from 155 mitochondria by TEV protease, the mRNA level of many mitochondrial proteins reduced (Figure 156 1C, Figure 1-figure supplement 1B). This transcription regulatory role of Tom70 is conserved 157 that an enhanced mitochondrial biogenesis was observed in fruit fly larvae when UAS-TOM70 
158

159

160

161

162

163

164

165

166

167

168

169

170

171

172

173

174

175

176

177

178

179

180

181

182

183

184

185

186

187

188

was overexpressed in muscles by Mef2-Gal4 (Figure 1C, Figure 1-figure supplement 1C). As overexpressing Tom70's cytosolic domain alone failed to activate the transcription of these mitochondrial proteins (Figure 1C), these results suggest that Tom70 levels on the mitochondrial outer membrane control the transcriptional activity of many mitochondrial proteins.

Which transcription factor(s) act downstream of Tom70? Retrograde signaling from mitochondria, marked by the increased nuclear-to-cytosol ratio of transcription factors $\mathrm{R} \operatorname{tg} 1 / 3$, is a known stress response towards mitochondrial stress and defects (Liu and Butow, 2006).

However, the nuclear/cytosol ratio of Rtg1/3-GFP in TOM70 OE cells is similar to the wild type cells cultured in the same galactose medium (Figure 1D, Figure 1-figure supplement 1D). Additionally, knocking out Rtg3 failed to prevent the activation of mitochondrial biogenesis program in TOM70 OE cells (Figure 1-figure supplement 1E). These results suggest that Tom70 regulates the transcription of mitochondrial proteins through transcription factors (TFs) other than the well-known retrograde signaling pathway. To search for the TFs downstream of Tom70, in silico analysis was conducted to find common TFs that recognize/bind the promoters of these mitochondrial genes (Monteiro et al., 2020). Some of these predicted TFs have expression changes upon TOM70 OE, indicating that Tom70 regulates these TFs (Figure 2A). A few of these TFs are so far not known to regulate mitochondrial biogenesis or metabolism (Figure 2B) (Bähler, 2005). For example, Fkh1/2 are members of the Forkhead family transcription factor that are previously known to regulate the expression of G2/M phase genes (Bähler, 2005; Martins et al., 2016; Postnikoff et al., 2012). Among these TFs that responded to Tom70 OE, overexpressing some TFs, including Fkh1/2, can mimic the TOM70 OE effect and increase the abundance of mitochondrial proteins from different sub-compartments, such as Adh3, Qcr7, and Tim44 (Figure 2C, D). We asked whether Fkh1/2 are required for the Tom70-related mitochondrial biogenesis program. When FKH1 or FKH2 are deleted, the induced expression of mitochondrial proteins in TOM70 OE cells is partially blocked (Figure 2E). We noticed that none of the Fkh1/2 mutants completely blocked the effect of TOM70 OE, indicating that Tom70 can signal through multiple TFs to regulate mitochondrial biogenesis. This is consistent with the observation that TOM70 OE has broader effects on mitochondrial biogenesis than OE of a single TF, such as HAP4 OE (Figure 1A). 


\section{Tom70 regulates the abundance of mtDNA}

191 Using single-photon sensitive APD and quantitative DNA staining (Gomes et al., 2018), we

192 found that in addition to the mitochondrial proteins, TOM70 OE also increased mtDNA 193 significantly (Figure 1D, 1E). In contrast to TOM70 OE, knocking out TOM70 led to the reduction of mtDNA (Figure 3A, B). Therefore, Tom70 regulates the biogenesis of both mitochondrial proteins and mtDNA. DNA polymerase $\gamma$ (Mip1 in yeast) is responsible for the replication and affects the abundance of mtDNA (Foury, 1989). We noticed that Mip1contains the iMTS which is known to use Tom70 for mitochondrial import (Figure 3C) (Backes et al., 2018). Indeed, the abundance of Mip1 protein was increased in TOM70 OE cells (Figure 3D), which likely contributes to the observed increase of mtDNA. Consistently, MIP1 OE increased the abundance of mtDNA (Figure 3E), albeit to a lesser extent than TOM70 OE (Figure 1E), suggesting that additional factors also contribute to the increase of mtDNA in TOM70 OE cells.

202 Interestingly, when mtDNA was removed, TOM70 OE could not activate the transcription of 203 these nDNA-encoded mitochondrial proteins ( $\rho^{0} ;$ TOM70 OE in Figure 1C). This suggests that 204 mtDNA is required for the Tom70-mediated transcriptional activation of nDNA-encoded 205 mitochondrial proteins.

\section{The Tom70-dependent regulation of mitochondrial biogenesis is involved in the cellular}

209 Our results indicate that Tom70 not only controls the import of mitochondrial proteins, but also

210 regulates their biogenesis. The nascent mitochondrial proteins synthesized in the cytosol are aggregation-prone and can cause cytosolic proteostasis stress and protein aggregation if not

212 imported in a timely manner (Nowicka et al., 2021; Wang and Chen, 2015; Wrobel et al.,

213 2015). It has been shown that impaired mitochondrial import triggers cellular responses to reduce

214 the biogenesis of mitochondrial proteins in order to achieve a new balance between biogenesis

215 and import of nascent mitochondrial proteins, alleviating their cytosolic accumulation and 216 aggregation (Samluk et al., 2018; Topf et al., 2019; Wrobel et al., 2015). Consistent with these

217 previous reports, no protein aggregation was detected when we blocked mitochondrial import by 218 shifting the $\operatorname{tim} 23^{t s}$ cells to the restricted temperature, which impaired the TIM complex (Figure 219 4A, C) (Pareek et al., 2013). 
221 Given Tom70's dual roles in mitochondrial import and transcriptional regulation, we considered

222 the possibility that Tom70 is involved in the cellular response to the impairment of mitochondrial

223 import (Figure 4A, B). To test if Tom70 is required for the repression of mitochondrial

224 biogenesis in $\operatorname{tim} 23^{t s}$ cells, we knocked out Tom70 and found that tim $23^{\text {ts }}$ cells lacking Tom70

225 formed large scale cytosolic protein aggregates upon Tim23 inactivation (Figure 4C). This

226 suggests that Tim23-inactivation signals through Tom70 to repress the biogenesis of

227 mitochondrial proteins and re-balance the biogenesis and import. As TOM70 OE upregulates

228 transcriptional activity of mitochondrial proteins, we tested whether overexpressing Tom 70 can

229 overwrite the repressive cellular program of mitochondrial biogenesis induced by tim $23^{\text {ts }}$ and

230 cause cytosolic aggregation. Indeed, when we overexpressed Tom70 upon tim $23^{\text {ts }}$ inactivation,

231 cells failed to maintain cytosolic homeostasis and showed large scale cytosolic protein

232 aggregation (Figure 4C). In contrast, TOM70 OE alone did not have this effect, suggesting that

the increase of mitochondrial biogenesis in TOM70 OE was balanced by enhanced mitochondrial

234 import capacity (Figure 4C). This is consistent with the increase of many TOM and TIM proteins

235 in TOM70 OE cells (Figure 1C). Next, we asked if this overwrite of repressive cellular program

236 and loss of balance between biogenesis-import is a general outcome of any pathways/factors that

237 induce mitochondrial biogenesis. However, HAP4 OE, which also controls mitochondrial

238 biogenesis, did not cause cytosolic protein aggregation upon tim $23^{\text {ts }}$, suggesting that the Tom70-

239 dependent mitochondrial biogenesis pathway plays a special role in the repressive cellular

240 program induced by tim $23^{t s}$. Together, these results are consistent with a model that Tom 70 is

241 involved in the cellular response to the impairment of mitochondrial import in tim $23^{\text {ts }}$ cells and

242 regulate the biogenesis of mitochondrial proteins (Figure 4-figure supplement 1) (Samluk et al.,

243 2018; Topf et al., 2019; Wrobel et al., 2015).

The reduction of Tom70 contributes to the age-related defects of mitochondria.

246 Tom70's transcription regulatory role led us to test whether Tom70 is a key factor in age-related

247 mitochondrial biogenesis defects, a conserved hallmark of aging (López-Otín et al., 2013).

248 Previous studies revealed that protein levels were increasingly uncoupled from their transcript

249 levels during aging (Janssens et al., 2015). Thus, we chose to follow protein, instead of mRNA,

250 abundance changes during aging to closely reflect the functional and physiological status of the 
251 aged cells. We examined different mitochondrial proteins in purified replicative old cells using

252 imaging and APDs, which allowed us to achieve single cell/age resolution, single-photon

253 sensitivity, and avoid the complications caused by the young cells contamination and impurity in

254 the purified old cells that were previously experienced in mass spectrometry-based studies

255 (Hendrickson et al., 2018; Janssens et al., 2015).

Using this approach, we found that Tom70 underwent age-dependent reduction (Figure 5A, B).

258 A similar level of Tom70 reduction was previously reported in aged rat (Kang et al., 2017). The reduction of Tom 70 is not unique as other mitochondrial proteins, including other TOM proteins, also showed age-dependent reduction (Figure 5B), which is consistent with the general trend of mitochondrial biogenesis defect observed in the previous proteomics study of aged cells (Janssens et al., 2015). This mitochondria biogenesis defect is associated with the loss of mitochondrial membrane potential, a common hallmark of mitochondrial defects (Figure 5C) (Hughes and Gottschling, 2012; Sun et al., 2016). To test if the reduction of Tom70 contributes to the loss of mitochondrial membrane potential in aged cells, we replaced TOM70's promoter with GAL promoter to prevent the age-associated reduction of Tom70. We found that overexpressing TOM70 can rescue mitochondrial membrane potential in aged cells (Figure 5C, D). This is unique to Tom70 as overexpressing other TOM proteins cannot fully prevent the loss of mitochondrial membrane potential during aging (Figure 5D). As mitochondrial membrane potential plays a key role in mitochondrial biogenesis, pGAL-TOM70 also prevented the ageassociated reduction of other mitochondrial proteins (Figure 5E, F).

These beneficial effects of TOM70 OE were not caused by the presence of galactose in the

274 medium as both control and TOM70 OE cells, as well as the cells overexpressing other TOM

275 proteins, were cultured in the same medium. In addition, similar results were observed when

276 Tom70 was expressed from the Z3EV promoter in the glucose medium (Figure 5-figure

277 supplement 1A, B) (McIsaac et al., 2013). In contrast, knocking out Tom70 accelerated the loss

278 of mitochondrial membrane potential and biogenesis defects of many other mitochondrial

279 proteins during aging (Figure 6A-D, Figure 6-figure supplement 1). Moreover, the $\Delta$ tom 70 cells

280 also lost mtDNA faster than wild type cells during aging (Figure 6E). This is likely due to the 281 accelerated loss of mtDNA polymerase Mip1, which uses Tom70 for import, in $\Delta$ tom 70 cells 
282

283

284

285

286

287

288

289

290

291

292

293

294

295

296

297

298

299

300

301

302

303

304

305

306

307

308

309

310

311

312

during aging (Figure 6C). The accelerated loss of mitochondrial biogenesis and functions likely explains previous results that knocking out Tom70 reduces the replicative lifespan (RLS) of yeast (Schleit et al., 2013). Consistent with this hypothesis, TOM70 OE, which activates mitochondrial biogenesis, can extend the RLS of budding yeast (Figure 6F). This RLS extension is similar to the caloric restriction (CR) of $0.1 \%$ glucose (Kaeberlein et al., 2004), which is known to increase the expression of many mitochondrial proteins (Lin et al., 2002; Ruetenik and Barrientos, 2015; Wuttke et al., 2012). Therefore, the reduction of Tom 70 contributes to the compromised mitochondrial biogenesis in aged cells.

It has been shown that CR can suppress the negative impact of many mitochondrial mutants, including $\Delta$ tom 70 , on the RLS of yeast (Schleit et al., 2013). For example, CR rescues the RLS of $\Delta P h b 1 / 2$ cells, the mitochondrial prohibitin complex required to stabilize newly imported proteins, by reducing the cytosolic translation which decreases the folding demands of nascent mitochondrial proteins and the load of proteostasis burden inside mitochondria (Schleit et al., 2013). As $\Delta$ tom 70 reduces the biogenesis/import of mitochondrial proteins during aging (Figure $6 \mathrm{~A}-\mathrm{E}$ ), which is different from the mitochondrial proteostasis defect caused by $\Delta p h b 1$, we considered a different mechanism that allows CR to rescue the RLS of $\Delta$ tom 70 cells (Figure 6G). Indeed, by checking mitochondrial proteins that showed accelerated biogenesis defects during the aging of $\Delta$ tom 70 cells, we found that their expression levels were rescued by $\mathrm{CR}$ during aging (Figure 6A-D, Figure 6-figure supplement 1). This suggests that CR restores the RLS of $\Delta t o m 70$ cells by preventing their premature mitochondrial biogenesis defect.

\section{Reduced biogenesis and enhanced degradation underlying the age-dependent reduction of mitochondrial Tom70.}

Age-related Tom70 reduction is likely a conserved process, as Tom70 protein is also reduced during fly aging and in aged or pathologically hypertrophic hearts from humans and rats (Kang et al., 2017; Li et al., 2014; Pacifico et al., 2018; Yang et al., 2019). In addition, overexpression of TOM70 was found to protect the cultured cardiomyocytes against diverse pro-hypertrophic insults (Kang et al., 2017; Li et al., 2014). Given these important roles of Tom70, we then asked what causes its age-associated reduction. The protein level of Tom70 is determined by its biogenesis and degradation. We first checked the biogenesis of Tom70 during aging. Changing 
313 TOM70's promoter prevented its age-dependent reduction, suggesting that the loss of

314 transcriptional activity likely contributes to the reduction of Tom70 during aging (Figure 5A, B

315 vs Figure 7-figure supplement 1A). Consistent with this hypothesis, analysis of the published

316 dataset revealed that the mRNA level of TOM70 is reduced in purified old yeast cells (Figure 7-

317 figure supplement 1B) (Janssens et al., 2015). To find TFs that regulate TOM70 expression, we

318 searched the database for TFs that recognize the promoter of TOM70 (Monteiro et al., 2020). We

319 confirmed that these predicted TFs either positively or negatively regulate Tom70-GFP level in

320 young cells (Figure 7-figure supplement 1C). Increasing the expression of some, but not all, TFs

321 is able to suppress the age-dependent reduction of Tom70 protein (Figure 7A). This suggests that

322 a reduced TF expression level, TF activity, the accessibility of promoters, or any of these factors

323 in combination, contributes to the reduction of Tom70 in aged cells (Hendrickson et al., 2018).

Many TFs, including the ones which regulate TOM70 expression, have low-complexity domains

326 (LCD) that are required for their transcriptional activity (Figure 7B, Figure 7-figure supplement

327 1D) (Alberti and Hyman, 2016; Boija et al., 2018; Sabari et al., 2018). The LCD-containing

328 proteins are conformationally flexible and sensitive to the proteostasis defect (Alberti and

329 Hyman, 2016; Liu et al., 2020; Mateju et al., 2017). Therefore, the loss of proteostasis, a

330 common aging hallmark, may reduce the stability and activity of these LCD-containing

331 transcription factors. Indeed, overexpression of chaperones prevented the reduction of Tom70 in

332 aged cells (Figure 7A). This is unlikely due to a direct upregulation of Tom 70 by chaperones as

333 they did not increase the Tom70 protein level in young cells, which do not have proteostasis

334 defect (Figure 7-figure supplement 1E). Although the detailed mechanisms await further

335 exploration, these results suggest that TOM70 expression and mitochondrial biogenesis is

336 impaired by the proteostasis defect in aged cells.

In addition to the biogenesis of Tom70, we asked whether aging affects the degradation of

339 Tom70 as well. Previous studies revealed that under acute vacuole deacidification Tom70 can be

340 sorted into mitochondria-derived compartment (MDCs) in a Fis1/Dnm1-dependent pathway for

341 degradation (Hughes et al., 2016). We asked whether Fis1 and Dnm1 also regulate Tom70

342 turnover during physiological aging. Indeed, cells lacking either Dnm1 or Fis 1 showed a

343 significantly higher expression level of Tom70 protein in both young (Figure 7-figure 
344 supplement 1F) and old cells (Figure 7C, D). Interestingly, the Dnm1 protein level increases with

345 age (Figure 7E, F), which could enhance the degradation of Tom70 and undermine the

346 biogenesis of mitochondria in aged cells.

\section{Discussion}

349 The results described above reveal a previously unknown role of Tom70 in regulating the

350 biogenesis of mitochondrial proteins. Cells use the same molecule, Tom70, to regulate the

351 transcriptional activity and import of mitochondrial proteins. These dual roles of Tom70 connect

352 two main steps of mitochondrial biogenesis that have been studied separately in the past, namely

353 the transcriptional regulations of mitochondrial proteins and the molecular mechanisms of

354 mitochondrial import. The imbalance between import and biogenesis of nascent mitochondrial

355 proteins leads to their cytosolic accumulation, which causes cytosolic proteostasis stress and the

356 formation of cytosolic protein aggregates (Samluk et al., 2018; Topf et al., 2019; Wrobel et al.,

357 2015). We propose that Tom70's dual roles in both biogenesis and import of mitochondrial

358 proteins allow the cells to accomplish mitochondrial biogenesis without compromising cytosolic

359 proteostasis (Figure 7G). This interdependence between cytosolic proteostasis and mitochondrial

360 biogenesis is consistent with the observations that mitochondrial dysfunction and protein

361 aggregation are two closely related hallmarks of aging and age-related diseases.

363 Retrograde signaling, such as the one mediated by Rtg1/2/3 in yeast and many forms of

364 mitochondrial unfolded protein response (UPR) (Shpilka and Haynes, 2018), has been described

365 for mitochondria to mitigate the proteostasis defect inside mitochondria. In contrast, this Tom70-

366 dependent transcriptional regulation of mitochondrial biogenesis is probably evolved to maintain

367 proteostasis outside of mitochondria. Consistent with this view, our results showed that this

368 Tom70-nucleus communication is independent of the classic retrograde signaling via Rtg1/2/3

369 that responds to the mitochondrial defects. Instead, several other TFs are required in this Tom70-

370 dependent transcriptional regulation of mitochondrial biogenesis. Some of these TFs, such as the

371 Forkhead family transcription factor Fkh1/2, could be the missing TFs that mediate the increase

372 of mitochondrial activity in response to certain longevity cues. For example, it was shown that

373 CR activates mitochondria biogenesis independent of Hap4, the well-studied TF of

374 mitochondrial biogenesis in yeast (Lin et al., 2002). Mining the published dataset, we found that 
375 Fkh1 is increased in CR-treated cells (Choi et al., 2017). The orthologs of these Forkhead family

376 transcription factors, such as Daf-16/FOXO, are integrators of different signal pathways to

377 regulate longevity and aging.

379 Our results also provide a new explanation for the mitochondrial defects in aged cells as the 380 reduction of Tom70 correlates with the loss of mitochondrial membrane potential, many 381 mitochondrial proteins, and mtDNA, which can be rescued by overexpressing Tom70.

382 Mechanistic dissection of this conserved Tom70 reduction in old cell suggests that age383 associated proteostasis defect, along with other factors, contribute to the reduction of Tom70.

384 Thus, Tom70 provides a novel connection between mitochondrial dysfunction and proteostasis 385 defects, two conserved hallmarks of aging. Many studies showed that loss of proteostasis is an 386 early event during aging (Ben-Zvi et al., 2009; Hipp et al., 2019; Yang et al., 2019). The loss of 387 proteostasis may inactivate some TFs required for the expression of Tom70 and other 388 mitochondrial proteins as many TFs contain low complex domains that are sensitive to proteostasis impairment (Alberti and Hyman, 2016; Boija et al., 2018; Liu et al., 2020; Vecchi et al., 2020). In addition, the increased level of Dnm1 during aging could further promote the degradation of mitochondrial Tom70. The reduction of Tom70 and mitochondrial biogenesis could lead to the previously observed age-dependent mitochondrial defects. The fact that loss of Tom70 accelerates aging and age-related mitochondrial defects, while TOM70 OE extends the RLS and delays these mitochondrial dysfunctions, highlights that Tom70 is a key molecule in mitochondrial aging.

\section{MATERIALS AND METHODS}

\section{Experimental Model Details}

All S. cerevisiae strains and plasmids used in this study are listed in the Key Resources Table

404 (supplemental table S1 and S3). All yeast strains used in this study are based on the BY4741

405 strain background. Genetic modifications were performed with PCR mediated homologous

406 recombination (Longtine et al., 1998) and genotyped with PCR to confirm correct modification

407 and lack of aneuploid for the chromosome that gene of interest is located. The GFP-tagged 
strains were from the GFP collection (Huh et al., 2003). The mutant strains were generated by PCR amplify KANMX cassette from existing mutant strains (Yeast Knockout Collection, GE Dharmacon) or pFA6a-KanMX plasmid to transform By4741 or the strains based on By4741. Expression of proteins from integration plasmids was done by integrating the linearized plasmid into TRP1 locus. For some strains, an empty integration plasmid was first integrated into TRP1 locus to provide the required sequences, such as AMP gene in the backbone of plasmid, for further integration of other plasmids. UAS-TOM70 fly was provided by Dr. Bingwei Lu (Gehrke et al., 2015) and crossed with Mef2-GAL4 to get 3rd instar larvae. UAS-TOM70 crossed with w1118 and Mef2-Gal4 crossed with w1118 served as genetic controls.

For confocal imaging, cells were grown at $30^{\circ} \mathrm{C}$ in $\mathrm{SC}$ glucose medium $(790 \mathrm{mg} / \mathrm{L}$ of complete supplement mixture (CSM) from Bioworld, $6.7 \mathrm{~g} / \mathrm{L}$ yeast nitrogen base, $2 \%$ glucose) and refreshed for an additional 2-3 hrs before imaging. For the strains with Gal promoter-driven proteins, cells were grown at $30^{\circ} \mathrm{C}$ in $\mathrm{SC} 2 \%$ raffinose medium overnight to $\mathrm{OD}_{600} \sim 0.5$ and induced by adding $2 \%$ galactose for 5 hrs. For qPCR assay, cells were cultured in YPD (2\% glucose, $1 \%$ yeast extract, $2 \%$ peptone), or YEPR (1\% yeast extract, $2 \%$ peptone, $2 \%$ raffinose) medium overnight and then induced with $2 \%$ galactose for $5 \mathrm{hrs}\left(\mathrm{OD}_{600}\right.$ between $\left.0.3-0.4\right)$ before harvest. Gal induction was ceased by adding $2 \%$ glucose for $30 \mathrm{~min}$ before other treatment or imaging. All media was prepared by autoclave the solution without glucose/raffinose/galactose for $20 \mathrm{~min}$ and adding filtered carbon source as indicated.

\section{Confocal microscopy} Images were acquired using a Carl Zeiss LSM-510 Confocor 3 system with 100x 1.45 NA PlanApochromat objective and a pinhole of one airy unit. The system was driven by Carl Zeiss AIM software for the LSM 510 meta. 405/488/561 nm laser was used to excite Calcofluor-white $(\mathrm{CFW}) / \mathrm{GFP} / \mathrm{RFPs}$, and emission was collected through the appropriate filters onto the single photon avalanche photodiodes on the Confocor 3. All CFW images were acquired through a 420 $\mathrm{nm}$ long pass filter, GFP images were acquired through a 505-540 nm filter, and RFP images were acquired with a 580-610 nm filter on the Confocor 3. All images were acquired in a multitrack, alternating excitation configuration so as to avoid bleed through. GFP-fusion strains were imaged with excitation laser at $8 \mathrm{~kW} / \mathrm{cm}^{2}$. Any strains used in comparison were acquired with the 
same laser and scanning setting to compare their expression levels. All image processing was performed in the Image $\mathrm{J}$ software (NIH, Bethesda, MD). The total amount of each protein (e.g. Tom70-GFP) or fluorescent dye (e.g. TMRM) was quantified from the sum projection of Z-

443 stacks after extracting the background signals. For visualization purposes, images scaled with 444 bilinear interpolation were used for figures.

\section{Drug treatment and key reagents}

447 Tetramethylrhodamine, Methyl Ester, Perchlorate (TMRM) (Thermo Fisher Scientific T668)

448 dissolved in DMSO to $1 \mathrm{mM}$ as stock solution, 1:10,000 for $15 \mathrm{~min}$ for mitochondrial membrane 449 potential detection and quantification. Calcofluor White Stain (Sigma-Aldrich 18909-100ML-F)

450 was diluted 200 folds in PBS and incubated with cells for $5 \mathrm{~min}$ at room temperature, followed 451 by three washes with PBS. Hoechst 33342 (Invitrogen ${ }^{\text {TM }} \mathrm{H} 3570$ ) $10 \mu \mathrm{g} / \mathrm{ml}$ was used to stain

452 DNA for $15 \mathrm{~min}$, followed by two washes with fresh medium before imaging. $\beta$-estradiol (Sigma 453 E8875) is used at $10 \mathrm{nM}$ or $100 \mathrm{nM}$ as final concentration to induce Tom70 overexpression from 454 Z3EV promoter.

\section{$R T-q P C R$}

457 For RT-qPCR assays, total RNA from yeast cells was isolated with TRIzol LS agent. Each RNA 458 sample $(1 \mu \mathrm{g})$ was subjected to reverse transcription (Invitrogen ${ }^{\mathrm{TM}}$ SuperScript ${ }^{\mathrm{TM}}$ III One-Step 459 RT-PCR System with Platinum ${ }^{\text {TM }}$ Taq DNA Polymerase, Invitrogen ${ }^{\mathrm{TM}}$ 12574018), and then 460 amplified by real-time PCR (Ssoadvanced ${ }^{\mathrm{TM}}$ Universal SYBR, bio-Rad 1725271). The primers 461 used for qPCR were shown in Supplementary Table S2. The relative values of gene expression 462 were calculated using the $2^{-\Delta \Delta C T}$ method (Livak and Schmittgen, 2001) by comparing the cycle 463 number for each sample with that for the untreated control. The results were normalized to the 464 expression level of tubulin or actin gene. All experiments have three independent replicates and 465 the mean was calculated for the figures. For Figure 1C, zy914 and zy930 were induced for 2 hrs 466 with 2\% galactose to express TEV protease and remove TOM70 cytosolic domain; zy1439, 467 zy1463, zy2974, zy2417, and zy3450 were induced for 5 hrs to overexpress Tom70 before 468 harvesting samples for mRNA extraction. 
469 For fly RT-qPCR, total RNA was extracted from $3^{\text {rd }}$ instar larvae (mixed sexes, 4 animals per

470 sample) with the same method described above for yeast. The primers used for fly qPCR were

471 shown in Supplementary Table S5.

472

\section{Isolation of Old Cells}

474 Yeast cells were collected from a fresh overnight culture and washed twice with cold PBS, $\mathrm{pH}$

475 8.0. About $4 \times 10^{7}$ mid-log cells were briefly concentrated by $3,000 \mathrm{~g}$ for $20 \mathrm{sec}$, followed by

476 three washes of cold sterile PBS pH 8.0. Cells were labeled with $1.6 \mathrm{mg} / \mathrm{ml}$ EZ-Link Sulfo-NHS-

477 LC-Biotin (Pierce) at room temperature in the same PBS for $30 \mathrm{~min}$ with gentle shaking. These

478 cells were used as M-cells. The M-cells were then washed three times with cold PBS, pH 7.2, to

479 get rid of free biotin. These M-cells were resuspended in $500 \mu \mathrm{PBS}$ pH 7.2 and mixed with $7 \mu 1$

480 BioMag magnetic streptavidin beads (Qiagen, 311714) for $60 \mathrm{~min}$ at $4^{\circ} \mathrm{C}$ and loaded into an

481 acrylic column with curved grade N52 magnet. A continuous flow of fresh medium was applied

482 at $0.3 \mathrm{ml} / \mathrm{min}$ to rinse away the daughter cells generated from these M-cells. After 60 hrs at room

483 temperature, the aged M-cells were unloaded from the device by removing the magnet and

484 flushed out with $3 \mathrm{ml} / \mathrm{min}$ fresh medium. The M-cells were then fixed with 1\% formaldehyde for

$48530 \mathrm{~min}$ at room temperature and washed three times with PBS, $\mathrm{pH}$ 7.4. After staining with CFW,

486 the cells were imaged with confocal to collect images of the bud scars and also the other

487 channels of interests. For live cell staining (e.g. TMRM staining), the old cells are stained

488 immediately after harvest without fixation. Cells with more than 12 bud scars were treated as old 489 cells.

490 While developing the old cells purification methodologies in lab, we noticed that environmental

491 stress, such as small amount of $\mathrm{NaOH} /$ ethanol left from sterilization step or the formation of

492 crowded cell clusters around the aging cells, caused the accumulation of Tom70-GFP in vesicles

493 for a fraction of old cells. Extensive overnight wash with fresh medium before loading cells into

494 device can eliminate this phenotype. This stress-induced age-dependent accumulation of Tom70-

495 GFP in vesicles for a portion of old cells could explain the phenotypes observed in a recent

496 report using microfluidics (Li et al., 2020). 
499

500

501

502

503

504

505

506

507

508

509

510

511

512

513

514

515

Replicative lifespan were examined as described previously (Steffen et al., 2009). Fresh YEP-2\% galactose (YEPG) plates were prepared one day before the assay. Fresh colonies from different strains were used to setup overnight culture in YPED and then spread 5 ul onto one side of the YEPG plates. Individual cells were aligned in the middle of plates with 20 cells per strain per plate. Both strains were included side-by-side with each other on each plate to reduce variations among plates. The plates were stored at $4{ }^{\circ} \mathrm{C}$ each night and kept at $30^{\circ} \mathrm{C}$ during the day. Each day the plates were kept at $30^{\circ} \mathrm{C}$ for about $10 \mathrm{hrs}$ during the dissections. The experiments were performed blindly by experimenter with the strain names and genotypes encoded by numbers.

\section{Predicted protein folding states}

Folding index(Lancaster et al., 2014) and intrinsic unstructured scores(Erdős and Dosztányi, 2020) were predicted using webservers:

\section{PLAAC: $\underline{\text { http://plaac.wi.mit.edu/ }}$}

\section{IUPred2a: https://iupred2a.elte.hu/}

\section{Statistical analysis}

All experiments were repeated multiple times to confirm reproducibility. Data are representative of at least three independent experiments. All quantifications are presented as the means \pm standard error of mean (s.e.m.). Statistical test for each bar graph in figures was determined by unpaired t test with Welch's correction in Prism 9. n.s. or ns, not significant; ${ }^{*} p<0.05 ;{ }^{*} p$ $<0.01 ; * * * p<0.001$. Mann-Whitney test was used for the RLS experiment.

\section{Acknowledgments}

The authors thank E.Verdin, G.Lithgow, and P.Walter for discussion. This work was supported by Glenn postdoctoral fellowship to Q.Liu, R01 AG058742 to B.K.Kennedy, and DP5OD024598 to C.Zhou.

\section{Author Contributions}

Conceptualization, Q.L. and C.Z.; Methodology, Q.L. and C.Z.; Investigation, Q.L., C.E.C., A.W., and C.Z.; Software, C.Z. and B.F.; Formal Analysis, C.Z., Q.L., and B.F.; Resource, C.Z., Q.L., B.K.K.; Writing - Original Draft, C.Z. and Q.L.; Writing - 
Review \& Editing, all authors; Funding Acquisition, Q.L., B.K.K., and C.Z.;

Supervision, C.Z.

\section{Declaration of Interests}

The authors declare no competing interests.

\section{DATA AVAILABILITY STATEMENT}

All plasmids and strains generated in this work can be requested from the corresponding author's

\section{Reference:}

Alberti, S., Hyman, A.A., 2016. Are aberrant phase transitions a driver of cellular aging? Bioessays 38, 959-968. doi:10.1002/bies.201600042

Backes, S., Hess, S., Boos, F., Woellhaf, M.W., Gödel, S., Jung, M., Mühlhaus, T., Herrmann, J.M., 2018. Tom70 enhances mitochondrial preprotein import efficiency by binding to internal targeting sequences. J. Cell Biol. 217, 1369-1382. doi:10.1083/jcb.201708044

Bähler, J., 2005. Cell-cycle control of gene expression in budding and fission yeast. Annu. Rev. Genet. 39, 69-94. doi:10.1146/annurev.genet.39.110304.095808

Baker, M.J., Tatsuta, T., Langer, T., 2011. Quality control of mitochondrial proteostasis. Cold Spring Harb. Perspect. Biol. 3. doi:10.1101/cshperspect.a007559

Ben-Zvi, A., Miller, E.A., Morimoto, R.I., 2009. Collapse of proteostasis represents an early molecular event in Caenorhabditis elegans aging. Proc. Natl. Acad. Sci. USA 106, 14914-14919. doi:10.1073/pnas.0902882106

Boija, A., Klein, I.A., Sabari, B.R., Dall'Agnese, A., Coffey, E.L., Zamudio, A.V., Li, C.H., Shrinivas, K., Manteiga, J.C., Hannett, N.M., Abraham, B.J., Afeyan, L.K., Guo, Y.E., Rimel, J.K., Fant, C.B., Schuijers, J., Lee, T.I., Taatjes, D.J., Young, R.A., 2018. Transcription Factors Activate Genes through the Phase-Separation Capacity of Their Activation Domains. Cell 175, 1842-1855.e16. doi:10.1016/j.cell.2018.10.042

Bruschini, C., Homulle, H., Antolovic, I.M., Burri, S., Charbon, E., 2019. Single-photon avalanche diode imagers in biophotonics: review and outlook. Light Sci Appl 8, 87. doi:10.1038/s41377-019-0191-5 
Choi, K.-M., Hong, S.-J., van Deursen, J.M., Kim, S., Kim, K.H., Lee, C.-K., 2017. Caloric restriction and rapamycin differentially alter energy metabolism in yeast. J. Gerontol. A, Biol. Sci. Med. Sci. 73, 29-38. doi:10.1093/gerona/glx024

de Winde, J.H., Grivell, L.A., 1993. Global regulation of mitochondrial biogenesis in Saccharomyces cerevisiae. Prog Nucleic Acid Res Mol Biol 46, 51-91. doi:10.1016/S0079-6603(08)61018-1

Drwesh, L., Rapaport, D., 2020. Biogenesis pathways of $\alpha$-helical mitochondrial outer membrane proteins. Biol. Chem. 401, 677-686. doi:10.1515/hsz-2019-0440

Erdős, G., Dosztányi, Z., 2020. Analyzing Protein Disorder with IUPred2A. Curr Protoc Bioinformatics 70, e99. doi:10.1002/cpbi.99

Forsburg, S.L., Guarente, L., 1989. Identification and characterization of HAP4: a third component of the CCAAT-bound HAP2/HAP3 heteromer. Genes Dev. 3, 1166-1178. doi:10.1101/gad.3.8.1166

Foury, F., 1989. Cloning and sequencing of the nuclear gene MIP1 encoding the catalytic subunit of the yeast mitochondrial DNA polymerase. J. Biol. Chem. 264, 20552-20560. doi:10.1016/S0021-9258(19)47098-1

Fox, T.D., 2012. Mitochondrial protein synthesis, import, and assembly. Genetics 192, 12031234. doi:10.1534/genetics.112.141267

Gehrke, S., Wu, Z., Klinkenberg, M., Sun, Y., Auburger, G., Guo, S., Lu, B., 2015. PINK1 and Parkin control localized translation of respiratory chain component mRNAs on mitochondria outer membrane. Cell Metab. 21, 95-108. doi:10.1016/j.cmet.2014.12.007

Glover, J.R., Lindquist, S., 1998. Hsp104, Hsp70, and Hsp40: a novel chaperone system that rescues previously aggregated proteins. Cell 94, 73-82. doi:10.1016/s00928674(00)81223-4

Gomes, C.J., Harman, M.W., Centuori, S.M., Wolgemuth, C.W., Martinez, J.D., 2018. Measuring DNA content in live cells by fluorescence microscopy. Cell Div. 13, 6 . doi:10.1186/s13008-018-0039-z

Gureev, A.P., Shaforostova, E.A., Popov, V.N., 2019. Regulation of Mitochondrial Biogenesis as a Way for Active Longevity: Interaction Between the Nrf2 and PGC-1 $\alpha$ Signaling Pathways. Front. Genet. 10, 435. doi:10.3389/fgene.2019.00435

Hendrickson, D.G., Soifer, I., Wranik, B.J., Kim, G., Robles, M., Gibney, P.A., McIsaac, R.S., 2018. A new experimental platform facilitates assessment of the transcriptional and chromatin landscapes of aging yeast. Elife 7. doi:10.7554/eLife.39911

Hipp, M.S., Kasturi, P., Hartl, F.U., 2019. The proteostasis network and its decline in ageing. Nat. Rev. Mol. Cell Biol. 20, 421-435. doi:10.1038/s41580-019-0101-y

Ho, B., Baryshnikova, A., Brown, G.W., 2018. Unification of Protein Abundance Datasets Yields a Quantitative Saccharomyces cerevisiae Proteome. Cell Syst. 6, 192-205.e3. doi:10.1016/j.cels.2017.12.004

Hoseini, H., Pandey, S., Jores, T., Schmitt, A., Franz-Wachtel, M., Macek, B., Buchner, J., Dimmer, K.S., Rapaport, D., 2016. The cytosolic cochaperone Sti1 is relevant for mitochondrial biogenesis and morphology. FEBS J. 283, 3338-3352. doi:10.1111/febs.13813

Hughes, A.L., Gottschling, D.E., 2012. An early age increase in vacuolar pH limits mitochondrial function and lifespan in yeast. Nature 492, 261-265. doi:10.1038/nature11654

Hughes, A.L., Hughes, C.E., Henderson, K.A., Yazvenko, N., Gottschling, D.E., 2016. Selective 
sorting and destruction of mitochondrial membrane proteins in aged yeast. Elife 5. doi:10.7554/eLife.13943

Huh, W.-K., Falvo, J.V., Gerke, L.C., Carroll, A.S., Howson, R.W., Weissman, J.S., O’Shea, E.K., 2003. Global analysis of protein localization in budding yeast. Nature 425, 686691. doi:10.1038/nature02026

Janssens, G.E., Meinema, A.C., González, J., Wolters, J.C., Schmidt, A., Guryev, V., Bischoff, R., Wit, E.C., Veenhoff, L.M., Heinemann, M., 2015. Protein biogenesis machinery is a driver of replicative aging in yeast. Elife 4, e08527. doi:10.7554/eLife.08527

Jornayvaz, F.R., Shulman, G.I., 2010. Regulation of mitochondrial biogenesis. Essays Biochem 47, 69-84. doi:10.1042/bse0470069

Kaeberlein, M., Kirkland, K.T., Fields, S., Kennedy, B.K., 2004. Sir2-independent life span extension by calorie restriction in yeast. PLoS Biol. 2, E296. doi:10.1371/journal.pbio.0020296

Kang, C., Qin, J., Osei, W., Hu, K., 2017. Regulation of protein kinase C-epsilon and its agedependence. Biochem. Biophys. Res. Commun. 482, 1201-1206. doi:10.1016/j.bbrc.2016.12.012

Kubitscheck, U., Kückmann, O., Kues, T., Peters, R., 2000. Imaging and tracking of single GFP molecules in solution. Biophys. J. 78, 2170-2179. doi:10.1016/S0006-3495(00)76764-6

Lancaster, A.K., Nutter-Upham, A., Lindquist, S., King, O.D., 2014. PLAAC: a web and command-line application to identify proteins with prion-like amino acid composition. Bioinformatics 30, 2501-2502. doi:10.1093/bioinformatics/btu310

Li, J., Qi, M., Li, C., Shi, D., Zhang, D., Xie, D., Yuan, T., Feng, J., Liu, Y., Liang, D., Xu, X., Chen, J., Xu, L., Zhang, H., Ye, J., Lv, F., Huang, J., Peng, L., Chen, Y.-H., 2014. Tom70 serves as a molecular switch to determine pathological cardiac hypertrophy. Cell Res. 24, 977-993. doi:10.1038/cr.2014.94

Li, Y., Jiang, Y., Paxman, J., O’Laughlin, R., Klepin, S., Zhu, Y., Pillus, L., Tsimring, L.S., Hasty, J., Hao, N., 2020. A programmable fate decision landscape underlies single-cell aging in yeast. Science 369, 325-329. doi:10.1126/science.aax9552

Lin, S.-J., Kaeberlein, M., Andalis, A.A., Sturtz, L.A., Defossez, P.-A., Culotta, V.C., Fink, G.R., Guarente, L., 2002. Calorie restriction extends Saccharomyces cerevisiae lifespan by increasing respiration. Nature 418, 344-348. doi:10.1038/nature00829

Liu, Z., Butow, R.A., 2006. Mitochondrial retrograde signaling. Annu. Rev. Genet. 40, 159-185. doi:10.1146/annurev.genet.40.110405.090613

Liu, Zhenying, Zhang, S., Gu, J., Tong, Y., Li, Yichen, Gui, X., Long, H., Wang, C., Zhao, C., Lu, J., He, L., Li, Ying, Liu, Zhijun, Li, D., Liu, C., 2020. Hsp27 chaperones FUS phase separation under the modulation of stress-induced phosphorylation. Nat. Struct. Mol. Biol. 27, 363-372. doi:10.1038/s41594-020-0399-3

Livak, K.J., Schmittgen, T.D., 2001. Analysis of relative gene expression data using real-time quantitative PCR and the 2(-Delta Delta C(T)) Method. Methods 25, 402-408. doi:10.1006/meth.2001.1262

Longtine, M.S., McKenzie, A., Demarini, D.J., Shah, N.G., Wach, A., Brachat, A., Philippsen, P., Pringle, J.R., 1998. Additional modules for versatile and economical PCR-based gene deletion and modification in Saccharomyces cerevisiae. Yeast 14, 953-961. doi:10.1002/(SICI)1097-0061(199807)14:10<953::AID-YEA293>3.0.CO;2-U

López-Otín, C., Blasco, M.A., Partridge, L., Serrano, M., Kroemer, G., 2013. The hallmarks of aging. Cell 153, 1194-1217. doi:10.1016/j.cell.2013.05.039 
Lowry, C.V., Zitomer, R.S., 1984. Oxygen regulation of anaerobic and aerobic genes mediated by a common factor in yeast. Proc. Natl. Acad. Sci. USA 81, 6129-6133. doi:10.1073/pnas.81.19.6129

Martins, R., Lithgow, G.J., Link, W., 2016. Long live FOXO: unraveling the role of FOXO proteins in aging and longevity. Aging Cell 15, 196-207. doi:10.1111/acel.12427

Mateju, D., Franzmann, T.M., Patel, A., Kopach, A., Boczek, E.E., Maharana, S., Lee, H.O., Carra, S., Hyman, A.A., Alberti, S., 2017. An aberrant phase transition of stress granules triggered by misfolded protein and prevented by chaperone function. EMBO J. 36, 16691687. doi:10.15252/embj.201695957

McIsaac, R.S., Oakes, B.L., Wang, X., Dummit, K.A., Botstein, D., Noyes, M.B., 2013. Synthetic gene expression perturbation systems with rapid, tunable, single-gene specificity in yeast. Nucleic Acids Res. 41, e57. doi:10.1093/nar/gks1313

McNabb, D.S., Xing, Y., Guarente, L., 1995. Cloning of yeast HAP5: a novel subunit of a heterotrimeric complex required for CCAAT binding. Genes Dev. 9, 47-58. doi:10.1101/gad.9.1.47

Monteiro, P.T., Oliveira, J., Pais, P., Antunes, M., Palma, M., Cavalheiro, M., Galocha, M., Godinho, C.P., Martins, L.C., Bourbon, N., Mota, M.N., Ribeiro, R.A., Viana, R., SáCorreia, I., Teixeira, M.C., 2020. YEASTRACT+: a portal for cross-species comparative genomics of transcription regulation in yeasts. Nucleic Acids Res. 48, D642-D649. doi:10.1093/nar/gkz859

Mootha, V.K., Bunkenborg, J., Olsen, J.V., Hjerrild, M., Wisniewski, J.R., Stahl, E., Bolouri, M.S., Ray, H.N., Sihag, S., Kamal, M., Patterson, N., Lander, E.S., Mann, M., 2003. Integrated analysis of protein composition, tissue diversity, and gene regulation in mouse mitochondria. Cell 115, 629-640. doi:10.1016/s0092-8674(03)00926-7

Morgenstern, M., Stiller, S.B., Lübbert, P., Peikert, C.D., Dannenmaier, S., Drepper, F., Weill, U., Höß, P., Feuerstein, R., Gebert, M., Bohnert, M., van der Laan, M., Schuldiner, M., Schütze, C., Oeljeklaus, S., Pfanner, N., Wiedemann, N., Warscheid, B., 2017. Definition of a High-Confidence Mitochondrial Proteome at Quantitative Scale. Cell Rep. 19, 28362852. doi:10.1016/j.celrep.2017.06.014

Newman, J.R.S., Ghaemmaghami, S., Ihmels, J., Breslow, D.K., Noble, M., DeRisi, J.L., Weissman, J.S., 2006. Single-cell proteomic analysis of S. cerevisiae reveals the architecture of biological noise. Nature 441, 840-846. doi:10.1038/nature04785

Nowicka, U., Chroscicki, P., Stroobants, K., Sladowska, M., Turek, M., Uszczynska-Ratajczak, B., Kundra, R., Goral, T., Perni, M., Dobson, C.M., Vendruscolo, M., Chacinska, A., 2021. Cytosolic aggregation of mitochondrial proteins disrupts cellular homeostasis by stimulating the aggregation of other proteins. Elife 10. doi:10.7554/eLife.65484

Olesen, J., Hahn, S., Guarente, L., 1987. Yeast HAP2 and HAP3 activators both bind to the CYC1 upstream activation site, UAS2, in an interdependent manner. Cell 51, 953-961. doi:10.1016/0092-8674(87)90582-4

Pacifico, R., MacMullen, C.M., Walkinshaw, E., Zhang, X., Davis, R.L., 2018. Brain transcriptome changes in the aging Drosophila melanogaster accompany olfactory memory performance deficits. PLoS One 13, e0209405. doi:10.1371/journal.pone.0209405

Pagliarini, D.J., Calvo, S.E., Chang, B., Sheth, S.A., Vafai, S.B., Ong, S.-E., Walford, G.A., Sugiana, C., Boneh, A., Chen, W.K., Hill, D.E., Vidal, M., Evans, J.G., Thorburn, D.R., Carr, S.A., Mootha, V.K., 2008. A mitochondrial protein compendium elucidates 
complex I disease biology. Cell 134, 112-123. doi:10.1016/j.cell.2008.06.016

Pareek, G., Krishnamoorthy, V., D'Silva, P., 2013. Molecular insights revealing interaction of Tim23 and channel subunits of presequence translocase. Mol. Cell. Biol. 33, 4641-4659. doi:10.1128/MCB.00876-13

Pickles, S., Vigié, P., Youle, R.J., 2018. Mitophagy and Quality Control Mechanisms in Mitochondrial Maintenance. Curr. Biol. 28, R170-R185. doi:10.1016/j.cub.2018.01.004

Pinkham, J.L., Guarente, L., 1985. Cloning and molecular analysis of the HAP2 locus: a global regulator of respiratory genes in Saccharomyces cerevisiae. Mol. Cell. Biol. 5, 34103416. doi:10.1128/mcb.5.12.3410

Postnikoff, S.D.L., Malo, M.E., Wong, B., Harkness, T.A.A., 2012. The yeast forkhead transcription factors fkh1 and fkh2 regulate lifespan and stress response together with the anaphase-promoting complex. PLoS Genet. 8, e1002583. doi:10.1371/journal.pgen.1002583

Prilusky, J., Felder, C.E., Zeev-Ben-Mordehai, T., Rydberg, E.H., Man, O., Beckmann, J.S., Silman, I., Sussman, J.L., 2005. FoldIndex: a simple tool to predict whether a given protein sequence is intrinsically unfolded. Bioinformatics $21,3435-3438$. doi:10.1093/bioinformatics/bti537

Ruetenik, A., Barrientos, A., 2015. Dietary restriction, mitochondrial function and aging: from yeast to humans. Biochim. Biophys. Acta 1847, 1434-1447. doi:10.1016/j.bbabio.2015.05.005

Sabari, B.R., Dall'Agnese, A., Boija, A., Klein, I.A., Coffey, E.L., Shrinivas, K., Abraham, B.J., Hannett, N.M., Zamudio, A.V., Manteiga, J.C., Li, C.H., Guo, Y.E., Day, D.S., Schuijers, J., Vasile, E., Malik, S., Hnisz, D., Lee, T.I., Cisse, I.I., Roeder, R.G., Sharp, P.A., Chakraborty, A.K., Young, R.A., 2018. Coactivator condensation at super-enhancers links phase separation and gene control. Science 361. doi:10.1126/science.aar3958

Samluk, L., Chroscicki, P., Chacinska, A., 2018. Mitochondrial protein import stress and signaling. Curr. Opin. Physiol. 3, 41-48. doi:10.1016/j.cophys.2018.02.010

Santangelo, G.M., 2006. Glucose signaling in Saccharomyces cerevisiae. Microbiol. Mol. Biol. Rev. 70, 253-282. doi:10.1128/MMBR.70.1.253-282.2006

Scarpulla, R.C., 2008. Transcriptional paradigms in mammalian mitochondrial biogenesis and function. Physiol. Rev. 88, 611-638. doi:10.1152/physrev.00025.2007

Schleit, J., Johnson, S.C., Bennett, C.F., Simko, M., Trongtham, N., Castanza, A., Hsieh, E.J., Moller, R.M., Wasko, B.M., Delaney, J.R., Sutphin, G.L., Carr, D., Murakami, C.J., Tocchi, A., Xian, B., Chen, W., Yu, T., Goswami, S., Higgins, S., Holmberg, M., Jeong, K.-S., Kim, J.R., Klum, S., Liao, E., Lin, M.S., Lo, W., Miller, H., Olsen, B., Peng, Z.J., Pollard, T., Pradeep, P., Pruett, D., Rai, D., Ros, V., Singh, M., Spector, B.L., Vander Wende, H., An, E.H., Fletcher, M., Jelic, M., Rabinovitch, P.S., MacCoss, M.J., Han, J.D.J., Kennedy, B.K., Kaeberlein, M., 2013. Molecular mechanisms underlying genotypedependent responses to dietary restriction. Aging Cell 12, 1050-1061. doi:10.1111/acel.12130

Schüller, H.-J., 2003. Transcriptional control of nonfermentative metabolism in the yeast Saccharomyces cerevisiae. Curr. Genet. 43, 139-160. doi:10.1007/s00294-003-0381-8

Shpilka, T., Haynes, C.M., 2018. The mitochondrial UPR: mechanisms, physiological functions and implications in ageing. Nat. Rev. Mol. Cell Biol. 19, 109-120. doi:10.1038/nrm.2017.110 
Sirrenberg, C., Bauer, M.F., Guiard, B., Neupert, W., Brunner, M., 1996. Import of carrier proteins into the mitochondrial inner membrane mediated by Tim22. Nature 384, 582 585. doi: $10.1038 / 384582 \mathrm{a} 0$

Steffen, K.K., Kennedy, B.K., Kaeberlein, M., 2009. Measuring replicative life span in the budding yeast. J. Vis. Exp. doi:10.3791/1209

Sun, N., Youle, R.J., Finkel, T., 2016. The mitochondrial basis of aging. Mol. Cell 61, 654-666. doi:10.1016/j.molcel.2016.01.028

Ter Linde, J.J.M., Steensma, H.Y., 2002. A microarray-assisted screen for potential Hap1 and Rox1 target genes in Saccharomyces cerevisiae. Yeast 19, 825-840. doi:10.1002/yea.879

Topf, U., Uszczynska-Ratajczak, B., Chacinska, A., 2019. Mitochondrial stress-dependent regulation of cellular protein synthesis. J. Cell Sci. 132. doi:10.1242/jcs.226258

Trueblood, C.E., Wright, R.M., Poyton, R.O., 1988. Differential regulation of the two genes encoding Saccharomyces cerevisiae cytochrome c oxidase subunit V by heme and the HAP2 and REO1 genes. Mol. Cell. Biol. 8, 4537-4540. doi:10.1128/mcb.8.10.4537

Vecchi, G., Sormanni, P., Mannini, B., Vandelli, A., Tartaglia, G.G., Dobson, C.M., Hartl, F.U., Vendruscolo, M., 2020. Proteome-wide observation of the phenomenon of life on the edge of solubility. Proc. Natl. Acad. Sci. USA 117, 1015-1020. doi:10.1073/pnas.1910444117

Vögtle, F.-N., Burkhart, J.M., Gonczarowska-Jorge, H., Kücükköse, C., Taskin, A.A., Kopczynski, D., Ahrends, R., Mossmann, D., Sickmann, A., Zahedi, R.P., Meisinger, C., 2017. Landscape of submitochondrial protein distribution. Nat. Commun. 8, 290. doi:10.1038/s41467-017-00359-0

Wang, X., Chen, X.J., 2015. A cytosolic network suppressing mitochondria-mediated proteostatic stress and cell death. Nature 524, 481-484. doi:10.1038/nature14859

Wiedemann, N., Pfanner, N., 2017. Mitochondrial machineries for protein import and assembly. Annu. Rev. Biochem. 86, 685-714. doi:10.1146/annurev-biochem-060815-014352

Wrobel, L., Topf, U., Bragoszewski, P., Wiese, S., Sztolsztener, M.E., Oeljeklaus, S., Varabyova, A., Lirski, M., Chroscicki, P., Mroczek, S., Januszewicz, E., Dziembowski, A., Koblowska, M., Warscheid, B., Chacinska, A., 2015. Mistargeted mitochondrial proteins activate a proteostatic response in the cytosol. Nature 524, 485-488. doi:10.1038/nature14951

Wuttke, D., Connor, R., Vora, C., Craig, T., Li, Y., Wood, S., Vasieva, O., Shmookler Reis, R., Tang, F., de Magalhães, J.P., 2012. Dissecting the gene network of dietary restriction to identify evolutionarily conserved pathways and new functional genes. PLoS Genet. 8, e1002834. doi:10.1371/journal.pgen.1002834

Yang, L., Cao, Y., Zhao, J., Fang, Y., Liu, N., Zhang, Y., 2019. Multidimensional Proteomics Identifies Declines in Protein Homeostasis and Mitochondria as Early Signals for Normal Aging and Age-associated Disease in Drosophila. Mol. Cell Proteomics 18, 2078-2088. doi:10.1074/mcp.RA119.001621

Young, J.C., Hoogenraad, N.J., Hartl, F.U., 2003. Molecular chaperones Hsp90 and Hsp70 deliver preproteins to the mitochondrial import receptor Tom70. Cell 112, 41-50. doi:10.1016/s0092-8674(02)01250-3

Zanphorlin, L.M., Lima, T.B., Wong, M.J., Balbuena, T.S., Minetti, C.A.S.A., Remeta, D.P., Young, J.C., Barbosa, L.R.S., Gozzo, F.C., Ramos, C.H.I., 2016. Heat Shock Protein 90 kDa (Hsp90) Has a Second Functional Interaction Site with the Mitochondrial Import Receptor Tom70. J. Biol. Chem. 291, 18620-18631. doi:10.1074/jbc.M115.710137 
bioRxiv preprint doi: https://doi.org/10.1101/2021.11.23.469672; this version posted November 23, 2021. The copyright holder for this preprint (which was not certified by peer review) is the author/funder, who has granted bioRxiv a license to display the preprint in perpetuity. It is made available under aCC-BY 4.0 International license.

802

803

804

805

806

807

808

809

810

\section{Main figure titles and legends} doi:10.1016/j.cell.2014.09.026

Zhou, C., Slaughter, B.D., Unruh, J.R., Guo, F., Yu, Z., Mickey, K., Narkar, A., Ross, R.T., McClain, M., Li, R., 2014. Organelle-based aggregation and retention of damaged proteins in asymmetrically dividing cells. Cell 159, 530-542. 
Figure 1 Over-expression

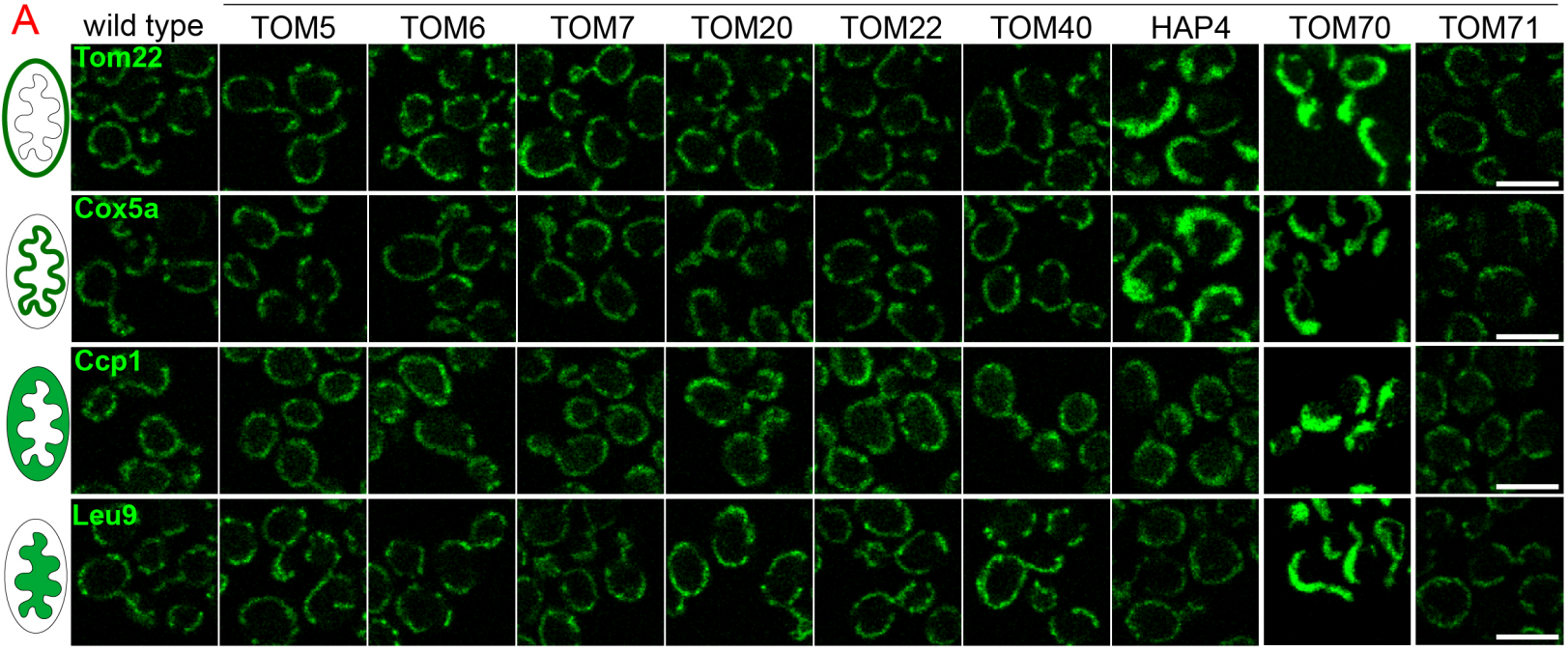

B

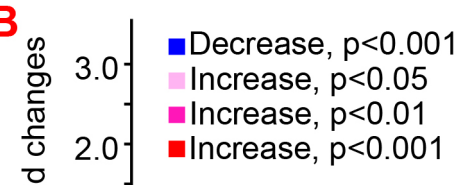

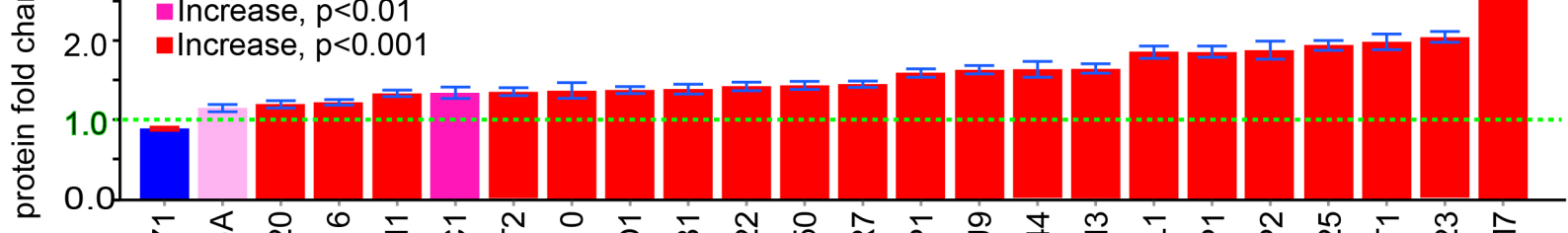

C

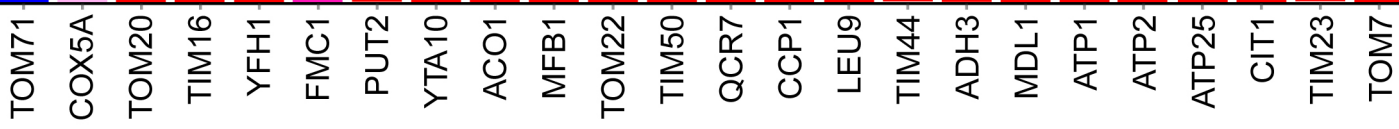

OTOM70 OE:
OTOM70 cyto. OE OTOM70 TEV $\bigcirc \boldsymbol{\rho}^{0}$; TOM70 OE T.y TOM70 OE

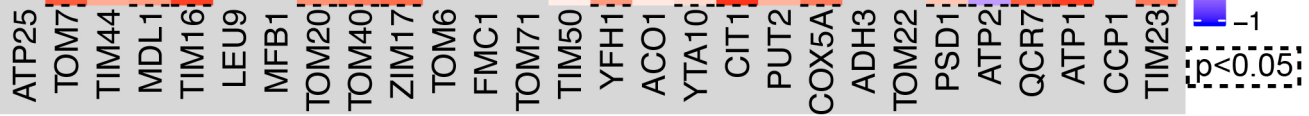

Dtg1-GFP
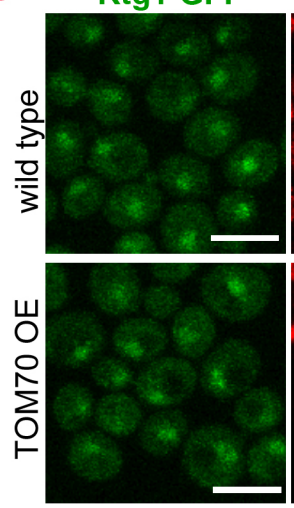

Mito.
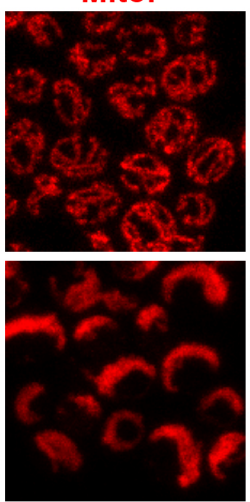

DNA

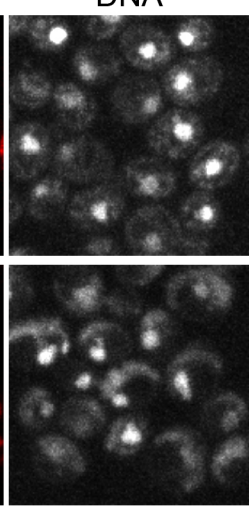

E

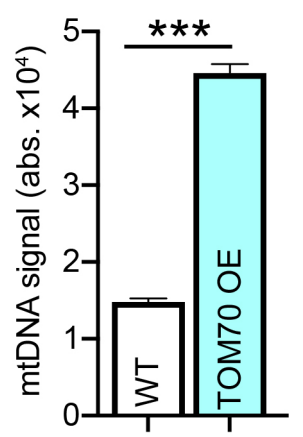

Figure 1: Tom70 regulates the transcriptional activity of mitochondrial proteins 
A. Representative images for proteins localized to different sub-compartments of mitochondria in cells overexpressing (OE) different TOM proteins and HAP4 from pGal promoter. HAP4 OE

817 increased the biogenesis of some, but not all, mitochondrial proteins. Mitochondrial proteins

818 were visualized by endogenous C-terminal GFP tagging and expressed from their native

819 promoters. As both wild type and OE strains were cultured in the same medium, the

820 mitochondrial biogenesis effect of TOM70 OE and HAP4 OE is not due to the presence of

821 galactose.

822 B. Quantification of mitochondrial protein levels in TOM70 OE cells normalized to wild type

823 cells cultured in the same medium. Mitochondrial proteins were visualized and quantified by

824 endogenous C-terminal GFP tagging. These mitochondrial proteins were randomly chosen from

825 the yeast GFP library based on their localization in mitochondria to represent all four sub-

826 compartments of mitochondria. Bar graphs are the normalized mean and s.e.m. Data were

827 analyzed with unpaired two-tailed $t$ test.

828 C. qPCR quantification of the mRNA abundance for different mitochondrial proteins in yeast

829 and fruit fly strains with different levels of mitochondrial Tom70. TOM70 cyto. OE,

830 overexpression of Tom70 cytosolic domain without transmembrane domain; TOM70 OE,

831 TOM70 overexpression; TOM70 TEV, the cytosolic domain of Tom70 was removed by TEV

832 protease. $\rho^{0}$, petite cells without mtDNA. For yeast, cells with the same genetic background but

833 lacking TOM70 TEV or TOM70 OE were used as control. For example, mRNA from $\rho^{0} / \mathrm{TOM} 70$

834 OE cells were normalized to $\rho^{0}$ cells lacking TOM70 OE. All different yeast strains, including

835 the controls, were cultured in the same galactose medium. For fruit fly, $3^{\text {rd }}$ instar larvae from

836 control (UAS-TOM70) and TOM70 OE (Mef2-Gal4; UAS-TOM70) were used to extract mRNA

837 from the whole animal. The mRNA abundance from each strain was normalized to control and

838 the average fold changes from three replicates are shown. Only conserved orthologous genes

839 from fly are included (www.alliancegenome.org). Dash line boxes indicate the ones with $\mathrm{p}<0.05$

840 from unpaired two-tailed t test.

841 D. Representative images of Rtg1-GFP in control and TOM70 OE cells. Rtg1-GFP, endogenous

842 C-terminal GFP tagging of Rtg1. DNA was stained with Hoechst dye. All different yeast strains

843 were cultured in the same galactose medium

844 E. Quantification of mtDNA abundance from Hoechst staining in (D). 1116 and 1090 cells

845 quantified for each. Data were analyzed with unpaired two-tailed $\mathrm{t}$ test: $* * *, \mathrm{p}<0.001$. 
bioRxiv preprint doi: https://doi.org/10.1101/2021.11.23.469672; this version posted November 23, 2021. The copyright holder for this preprint (which was not certified by peer review) is the author/funder, who has granted bioRxiv a license to display the preprint in perpetuity. It is made available under aCC-BY 4.0 International license.

846 Scale bar for all images: $5 \mu \mathrm{m}$. Images are representative of at least two independent

847 experiments. Sample sizes in B are given in Table S4.

848

849

850 
Figure 2

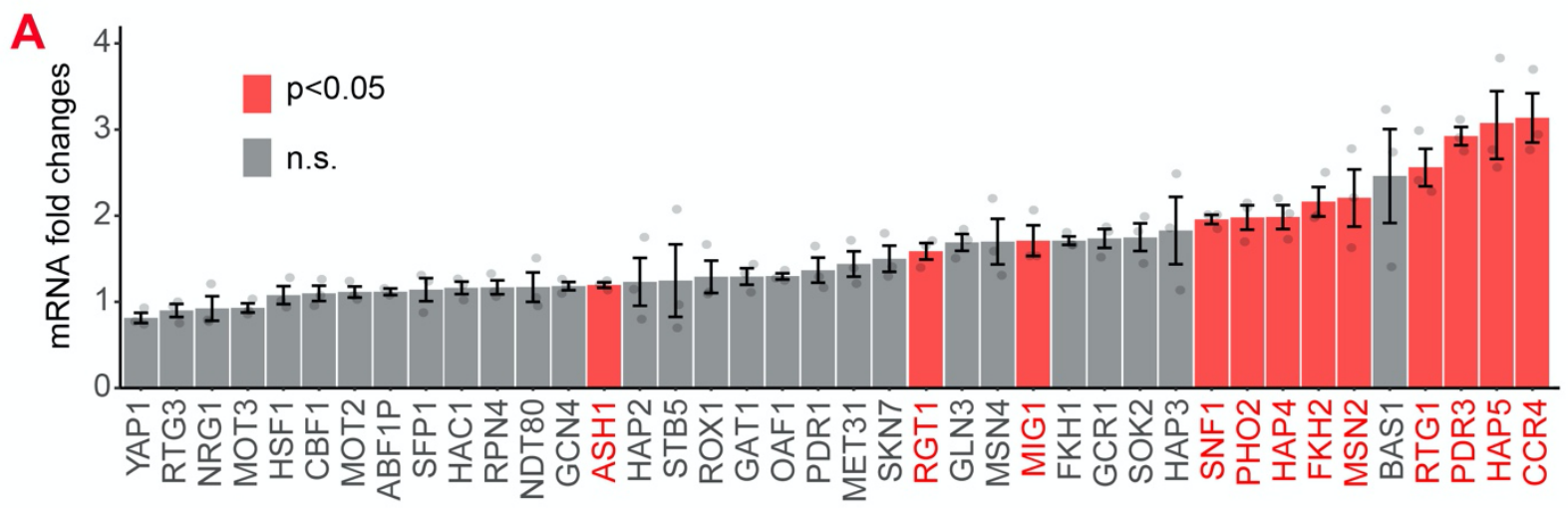

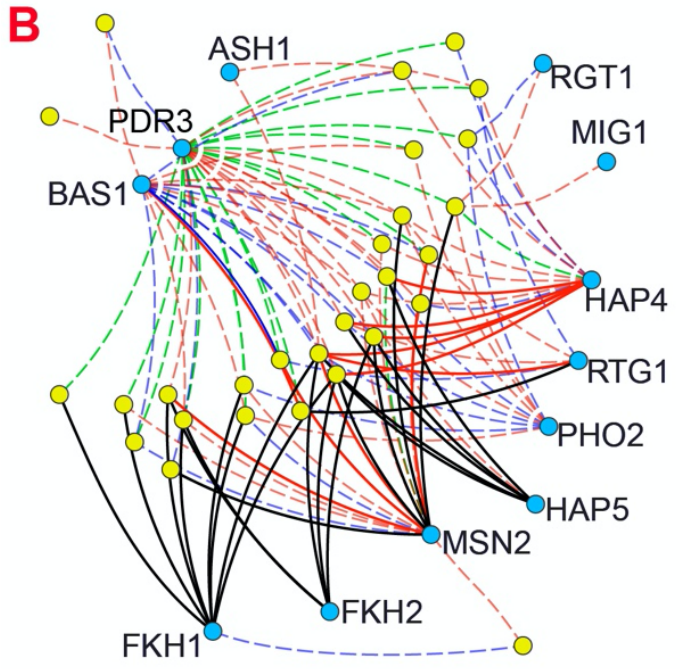

Mitochondrial proteins

$\begin{array}{ll}--- \text { Positive } & --- \text { Positive\&Negative } \\ ---- \text { Negative } & \text { Unknown }\end{array}$
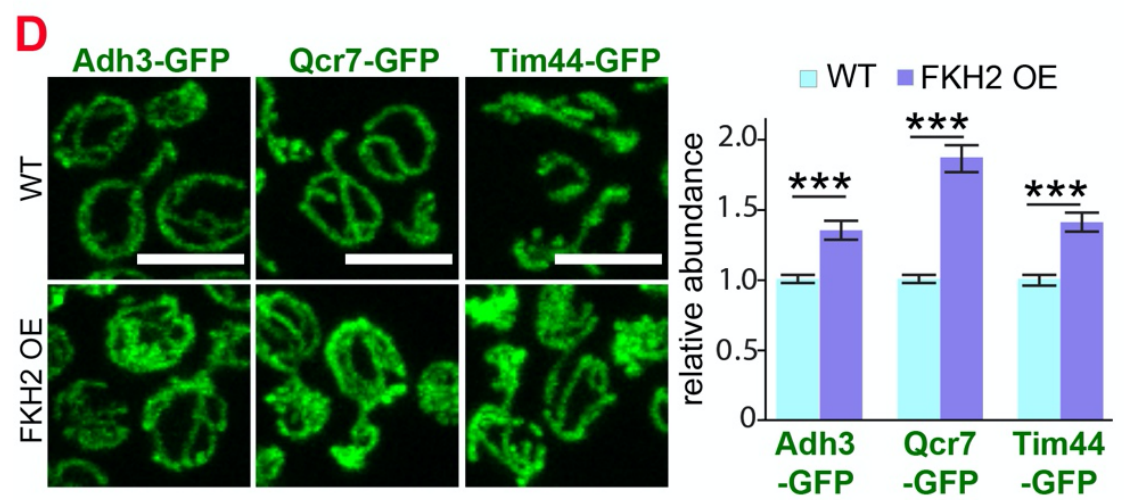

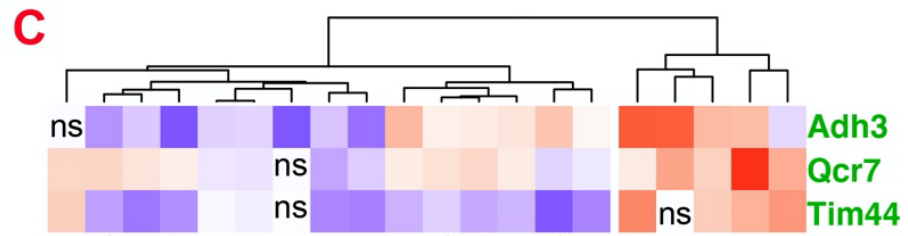

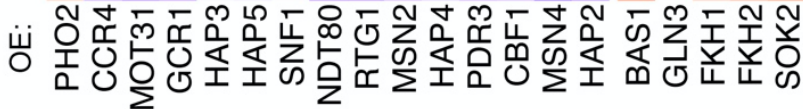

protein fold changes

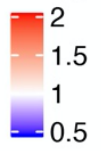

$\mathbf{E}$
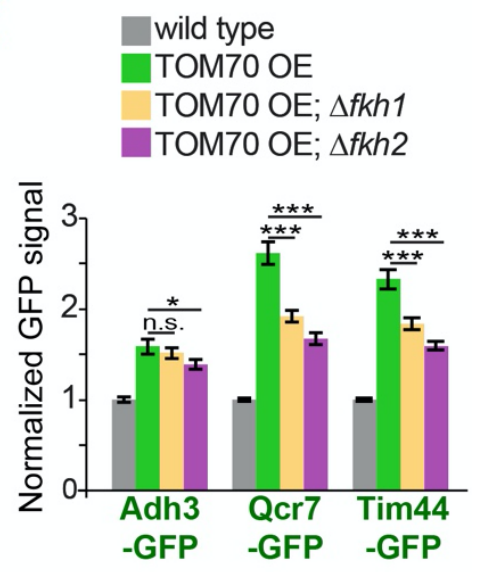

852 Figure 2: The transcription factors involved in the Tom70-dependent transcriptional

853 regulation of mitochondrial proteins. 
A. mRNA abundance of different transcription factors (TF) quantified by qPCR in TOM70 OE strain and normalized to control cells. All different yeast strains, including wild type control, were cultured in the same medium. Shown are the mean and s.e.m. from three replicates. B. TF regulatory network predicted for these mitochondrial proteins tested in Figure 1B (Monteiro et al., 2020). Only the TFs that increase significantly in (A) is included to simplify the illustration. The positive and negative regulations from existing literature are shown with red and blue lines, respectively; green lines represent the ones have both positive and negative effects. Solid lines represent the DNA binding evidence and the dash lines for co-expression evidence.

862 The predicted regulations based on the consensus sequences but have no previous experimental 863 data are shown as black lines.

864 C. Expression changes of mitochondrial proteins from different sub-compartments upon overexpression of different TFs. The GFP signal of Adh3-GFP, Qcr7-GFP, and Tim44-GFP were quantified from TF OE cells and normalized to wild type control of each protein. $p<0.05$

867 for all of them except for four that labeled as 'ns'. Data were analyzed with unpaired two-tailed t test. Both control and TOM70 OE strains were cultured in SC-raffinose medium and added 2\%

869 galactose for 5 hours before sample collection.

870 D. Representative images and quantification of different mitochondrial proteins in wild type and

871 FKH2 OE strain. All different yeast strains were cultured in the same medium.

872 E. FKH1 and FKH2 are partially required for the mitochondria biogenesis program downstream 873 of TOM70 OE. GFP signal of mitochondrial proteins were quantified in different strains and 874 normalized to wild type.

875 Scale bar for all images: $5 \mu \mathrm{m}$. Mitochondrial proteins were visualized by endogenous C-

876 terminal GFP tagging and expressed from their own promoters. Images are representative of at

877 least two independent experiments. Bar graphs are the normalized mean and s.e.m. Data were 878 analyzed with unpaired two-tailed t test: $* * *, p<0.001 ; *, p<0.05$; n.s., not significant. Sample 879 sizes in $(\mathrm{D}, \mathrm{E})$ are given in Table S4. 


\section{Figure 3}
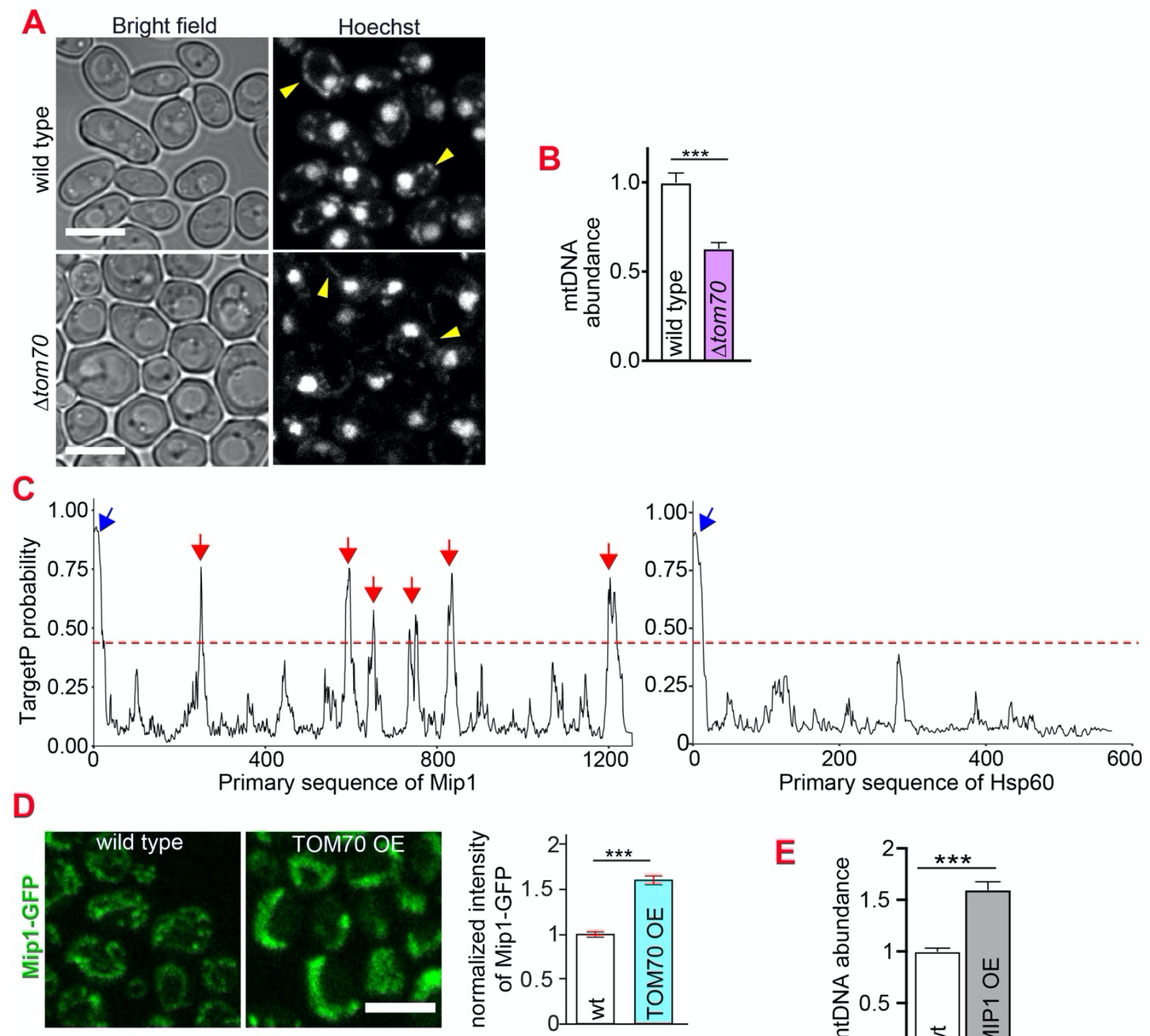

Figure 3: Tom70 regulates the abundance of mtDNA

886 A, B. Representative images and quantification of mtDNA in wild type and $\Delta$ tom 70 cells were

887 stained by Hoechst dye. Yellow arrowheads point to the mtDNA. Bar graph are the mean and

888 s.e.m. from 87 and 109 cells.

889 C. iMTS probability profiles of Mip1 and Hsp60 predicted by TargetP algorithm. The

890 consecutively N-terminally truncated sequences of the proteins were used as input to calculate

891 the TargetP scores for each residue, which shows internal regions with presequence-like

892 properties, or iMTS. Hsp60, another mitochondrial protein does not depend on Tom70, was also

893 plotted in the same way for comparison (Backes et al., 2018). Red line (0.4) indicates the cutoff 
894 defined in previous study (Backes et al., 2018). Blue and red arrows indicate the N-terminal

895 signal peptide and iMTS, respectively.

896 D. Representative images and quantification of Mip1-GFP in control and TOM70 OE cells. Bar

897 graph are the mean and s.e.m. from 300 and 438 cells.

898 E. Quantification of mtDNA in control and MIP1 OE cells from Hoechst staining. Bar graph are

899 the mean and s.e.m. from 253 and 209 cells.

900 Scale bar for all images: $5 \mu \mathrm{m}$. Images are representative of at least two independent

901 experiments. Bar graphs are the normalized mean and s.e.m. Data were analyzed with unpaired

902 two-tailed t test: $* * *, \mathrm{p}<0.001$.

903

904

905

906

907

908

909

910

911

912

913

914

915

916

917

918

919

920

921

922

923

924 


\section{Figure 4}
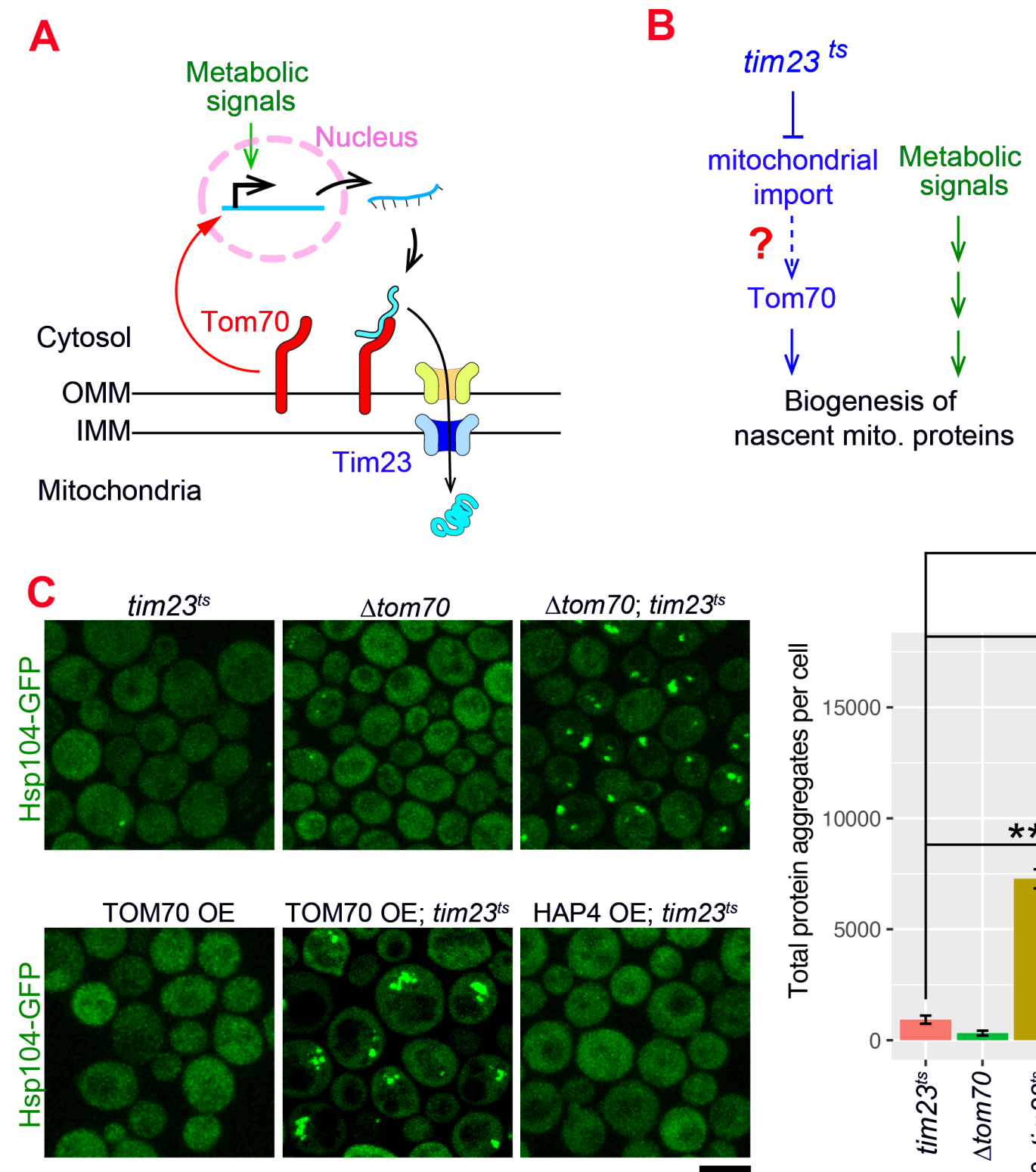

\section{Figure 4: The Tom70-dependent regulation of mitochondrial biogenesis is involved in the}

927 cellular response to the mitochondrial import defect.

928 A. Mitochondrial biogenesis in wild type cells. Metabolic signals regulate the transcriptional

929 activity of mitochondrial proteins, which are synthesized in the cytosol and translocated through

930 the outer mitochondrial membrane (OMM) and inner mitochondrial membrane (IMM) via TOM-

931 TIM complexes. Tom70, a key component of the TOM complex, serves as the import receptor 
932 and the biogenesis regulator of mitochondrial proteins. Tim23, a key component of the TIM

933 complex, acts downstream and receives the incoming nascent mitochondrial proteins from the

934 TOM complex.

935 B. The potential role of Tom70 in the cellular response to the impairment of mitochondrial

936 import caused by tim $23^{t s}$.

937 C. Representative images and quantification for different strains after switching to the restricted 938 temperatures to inactivate $\operatorname{tim} 23^{\text {ts }}$. All strains were cultured in raffinose medium at $25^{\circ} \mathrm{C}$

939 overnight, followed by adding galactose for $3 \mathrm{hrs}$ before adding glucose for 30min and switching

940 to $35^{\circ} \mathrm{C}$ for $2 \mathrm{hrs}$ to inactivate $\operatorname{tim} 23^{\text {ts }}$. Cytosolic protein aggregation was visualized with GFP-

941 tagged Hsp104, a general marker of protein aggregates (Glover and Lindquist, 1998; Zhou et al.,

942 2014). Bar graphs are the mean and s.e.m. of the Hsp104-GFP signals inside protein aggregates

943 in each cell. Scale bar: $5 \mu \mathrm{m}$. Images are representative of at least two independent experiments.

944 Data were analyzed with unpaired two-tailed t test: ***, $\mathrm{p}<0.001$; n.s., not significant. Sample

945 sizes in (C) are given in Table S4. 
Figure 5
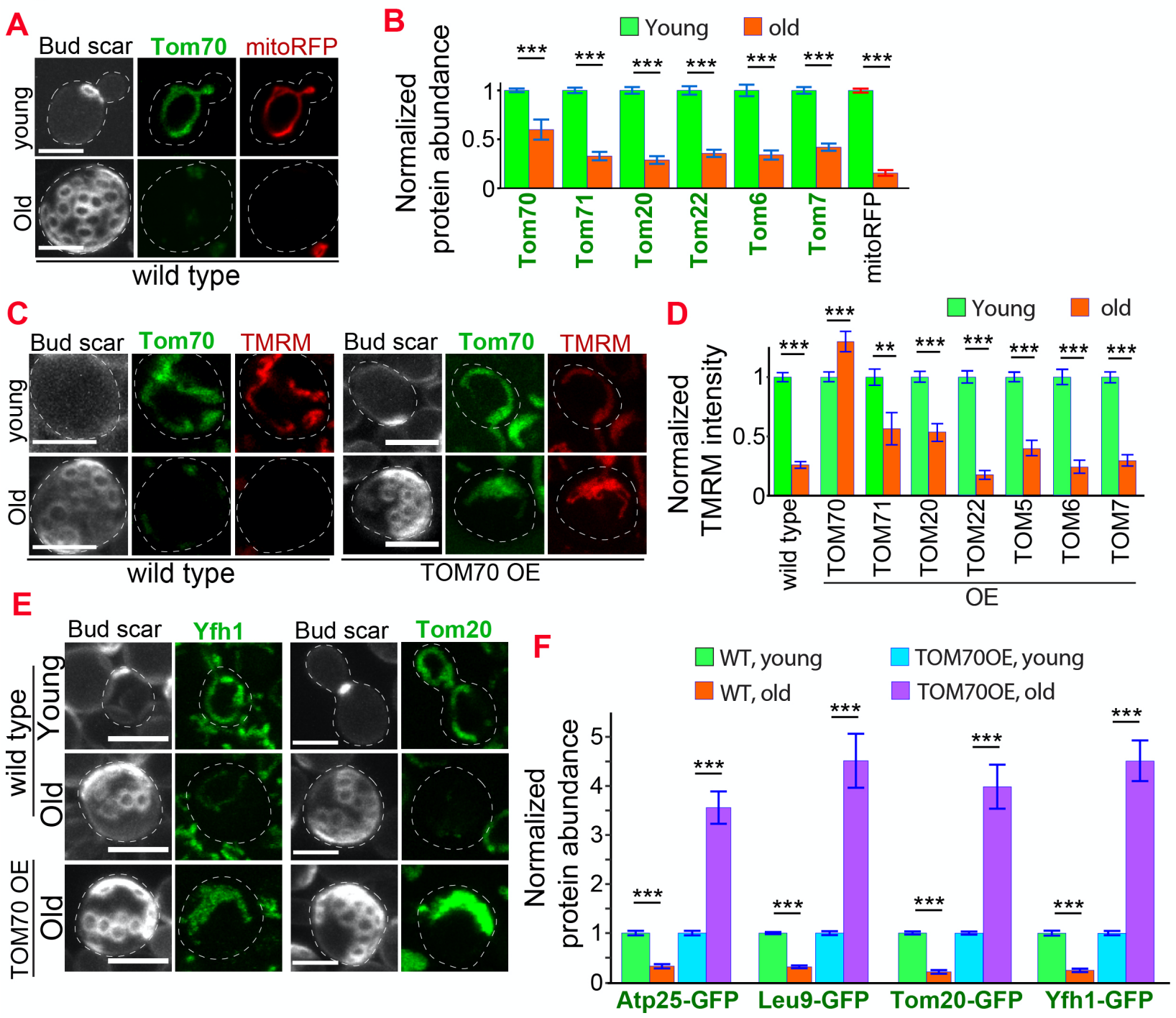

Figure 5: Age-related reduction of Tom70 is associated with mitochondrial dysfunctions.

961 A. Representative images of Tom70-GFP and mito-RFP in young and aged cells. Both young

962 and old cells were from YPD culture and stained with calcofluor white to visualize the bud scars.

963 B. Quantification of different GFP-tagged TOM proteins and mitoRFP in young and aged cells.

964 Both young and old cells were from YPD culture and the GFP signal was normalized to

965 corresponding young cells of the same strain.

966 C, D. Representative images (C) and quantification (D) of mitochondrial membrane potential in

967 young and aged cells from different strains. Different TOM proteins were overexpressed from

968 pGAL promoter. Both young and old cells were from YEP-galactose medium. Mitochondrial

969 membrane potential was indicated by TMRM staining. 
970 E, F. Representative images (E) and quantification (F) of mitochondrial proteins undergo age-

971 associated loss of expression, which can be suppressed by overexpressing TOM70. WT, wild

972 type. Both young and old cells were from YEP-galactose medium.

973 Scale bar for all images: $5 \mu \mathrm{m}$. Mitochondrial proteins were visualized by endogenous C-

974 terminal GFP tagging and expressed from their own promoters. Both young and old cells in each

975 experiment went through the same culture medium, experimental procedures, and purification.

976 Images are representative of at least two independent experiments. Bar graphs are the mean and

977 s.e.m. that normalized to young cells for each genotype. Sample sizes of (B, D, F) are given in

978 Table S4. Data were analyzed with unpaired two-tailed t test: ***, p $<0.001 ; * *, p<0.01 ; *$,

$979 \mathrm{p}<0.05$. 
Figure 6

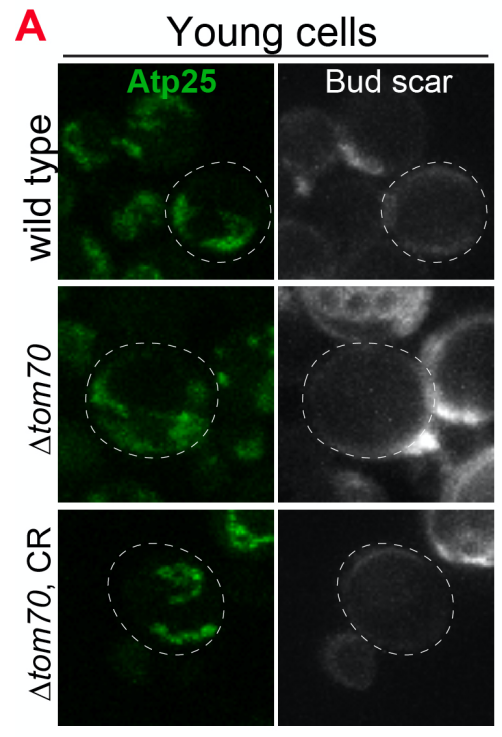

C

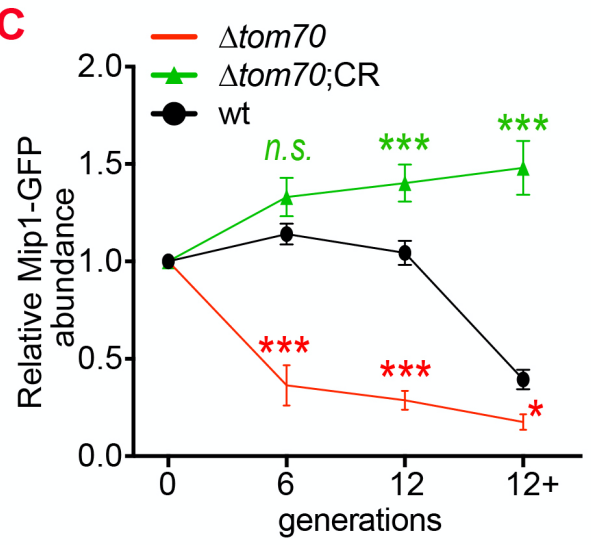

F
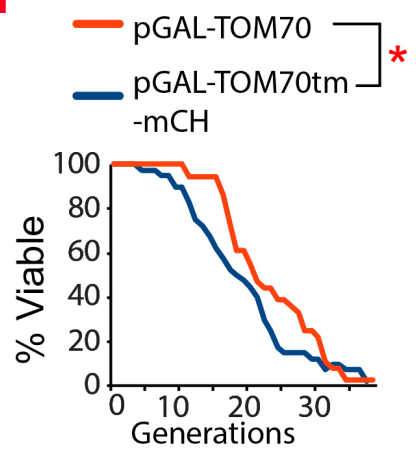

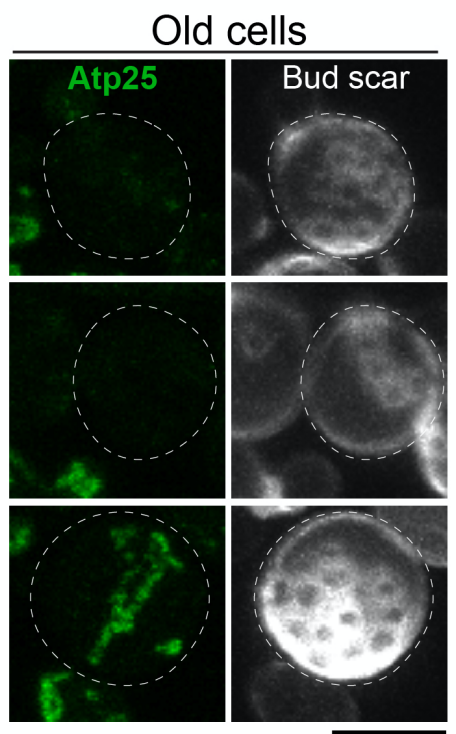

D

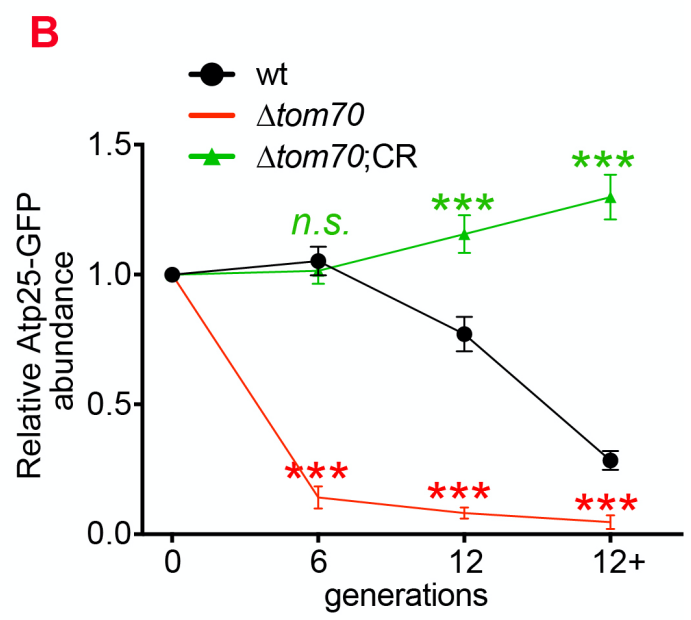

E
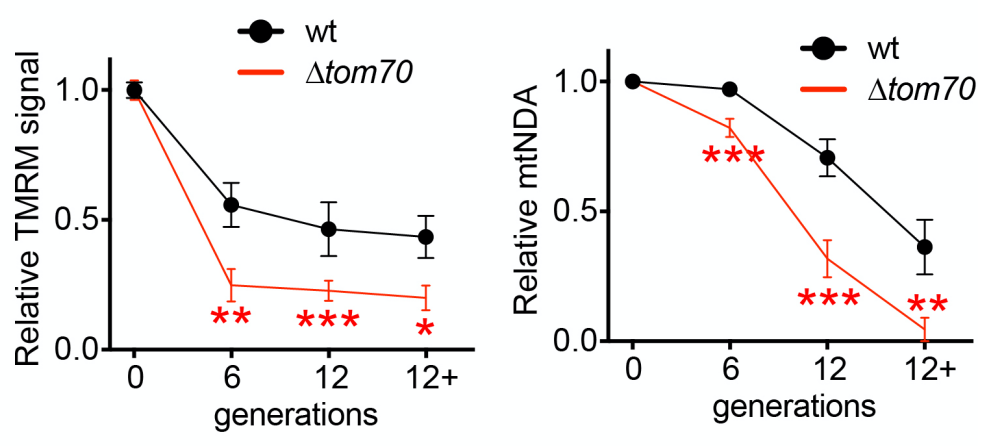

G

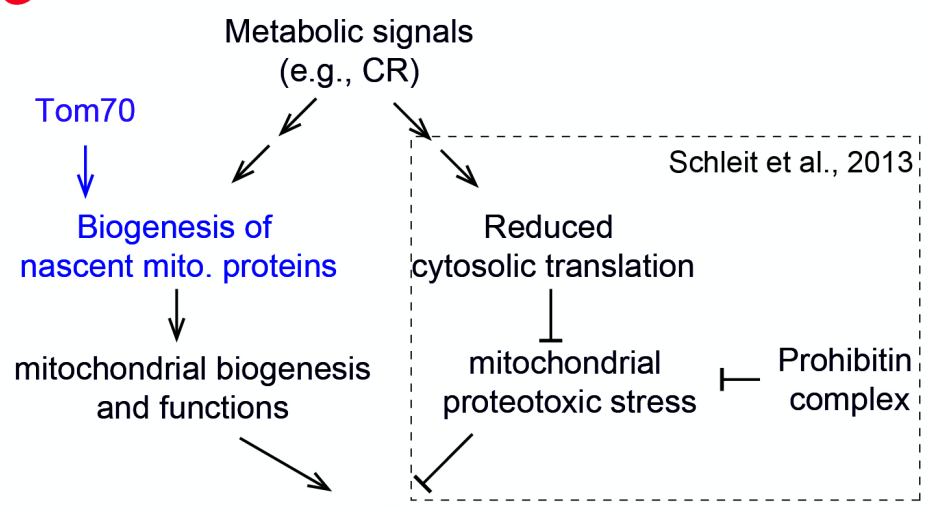

RLS

1002 Figure 6: Loss of Tom70 accelerates mitochondrial aging.

1003 A, B. Representative images (A) and quantifications (B) of Atp25-GFP in young and aged cells 1004 of different strains. Both young and old cells were from YPD culture and stained with calcofluor 1005 white to visualize the bud scars. Both young and old cells were quantified from the purified cells 
1006

1007

1008

1009

1010

1011

1012

1013

1014

1015

1016

1017 glucose.

1018

1019

1020

1021

1022

1023

1024

1025

1026

1027

1028

1029

1030

1031

1032

1033

1034

that went through the same experimental procedures and purification (same for other results). All strains cultured in YPD medium with $2 \%$ glucose except for CR, which has $0.05 \%$ glucose. The GFP signal quantified from the old cells was normalized to corresponding young cells of the same strain. Old cells were grouped as young ( 0 bud scar), age 2-6, age $7-11$, and age $12+$. Data were analyzed with unpaired two-tailed t test: ${ }^{* * *}, \mathrm{p}<0.001 ;{ }^{*}, \mathrm{p}<0.01 ; *, \mathrm{p}<0.05 ;$ n.s., not significant (same for C-E). More than 20 cells quantified for each group (same for C-E).

C. Quantification of Mip1 in young and aged cells from different strains. All strains cultured in YPD medium with $2 \%$ glucose except for CR, which has $0.05 \%$ glucose.

D, E. Quantification of mitochondrial membrane potential (D) and mtDNA (E) in young and aged cells from different strains. Mitochondrial membrane potential was indicated by TMRM staining. All strains cultured in YPD medium with 2\% glucose except for CR, which has $0.05 \%$

F. Survival curve from replicative life span assay of 36 and 40 cells expressing pGAL-TOM70 and pGAL-TOM70tm-mCH, respectively, were determined by microscopic dissection on YEPgalactose plates. $\mathrm{p}=0.038$ by Mann-Whitney test.

G. Proposed model by which CR extends the RLS of different mitochondrial mutants via different mechanisms. Schleit et al., 2013 showed that CR extends the RLS of $\Delta p h b$ cells by reducing mitochondrial proteotoxic stress via reduced cytosolic translation. Our results demonstrated that this reduced cytosolic translation in CR is not universal as the biogenesis of mitochondrial proteins is preserved during aging. This preserved mitochondrial biogenesis in CR is consistent with the observed rescue of RLS in $\Delta$ tom 70 cells by CR.

Scale bar for all images: $5 \mu \mathrm{m}$. Mitochondrial proteins were visualized by endogenous Cterminal GFP tagging and expressed from their own promoters. Images are representative of at least two independent experiments. 
Figure 7

A

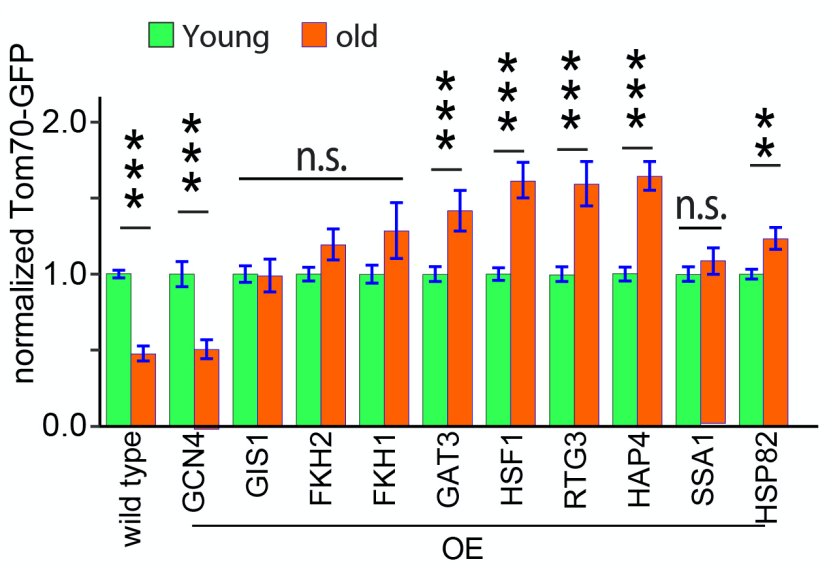

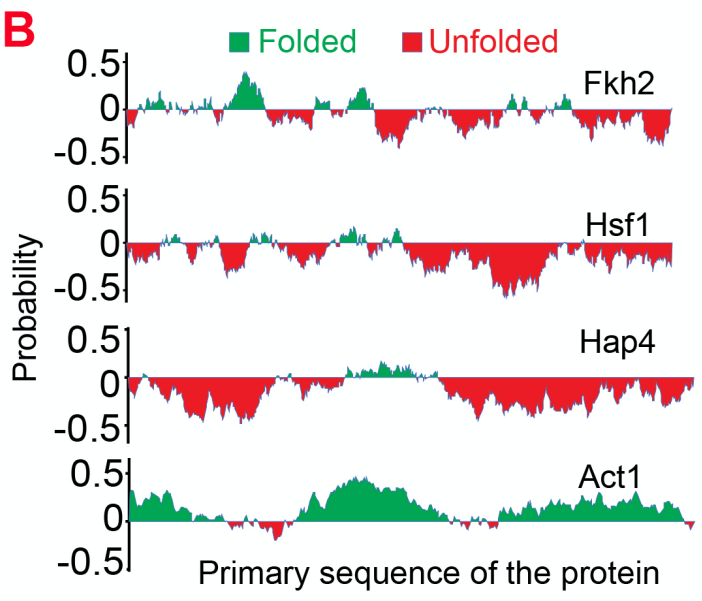

$\Delta$ fis1

C
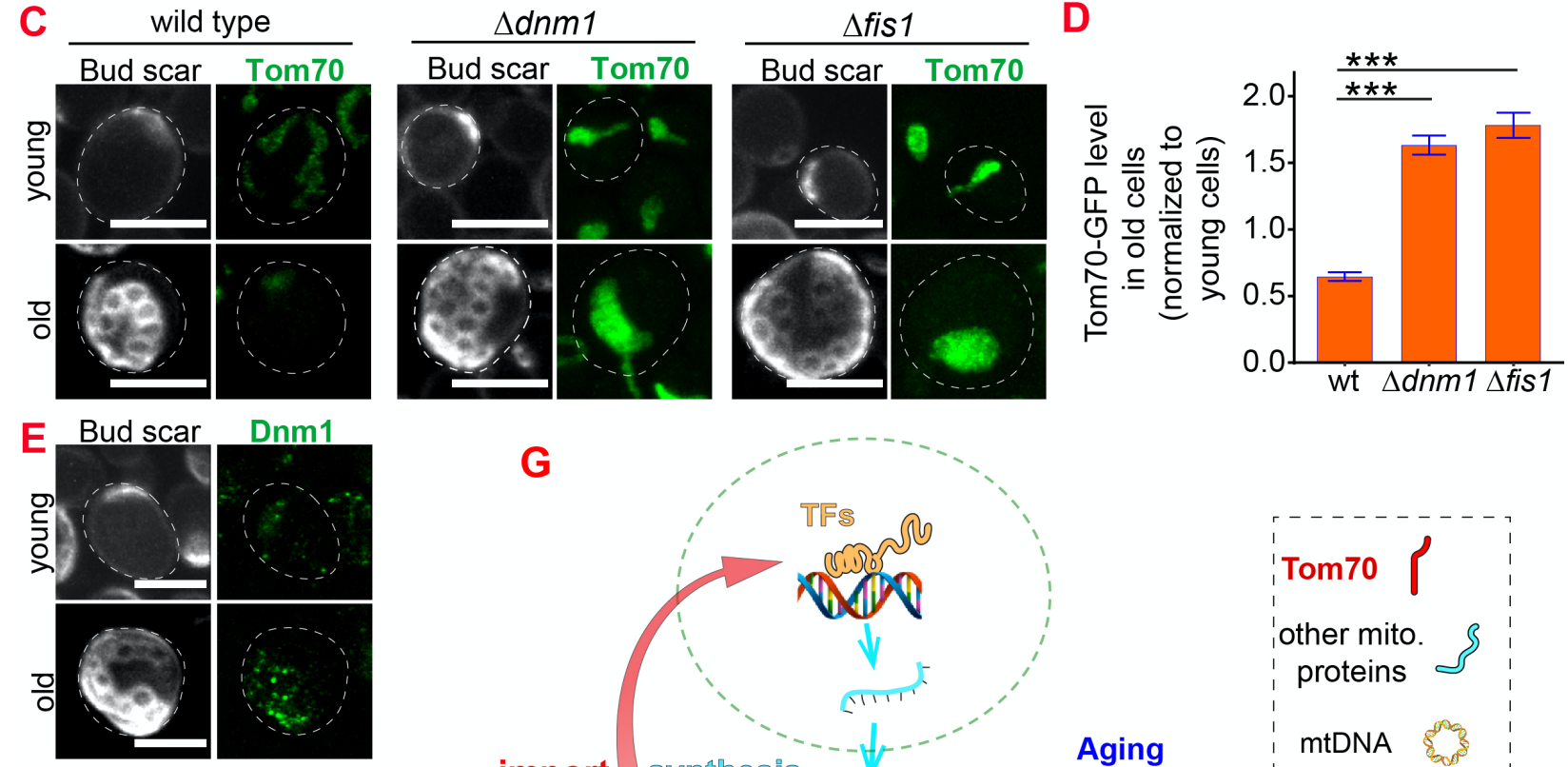

$\mathbf{F}$

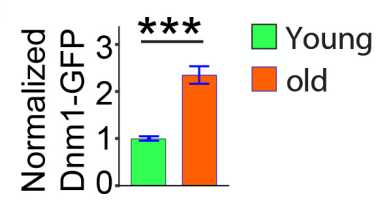

Figure 7: Reduced biogenesis and enhanced degradation underlying the age-dependent 
1038 A. Mean and s.e.m. of Tom70-GFP signal in young and old cells from strains overexpressing 1039 different TFs and chaperones. Sample sizes are given in Table S4. Both young and old cells were 1040 from YEP-galactose medium.

1041 B. Predicted folding index of different proteins (Erdős and Dosztányi, 2020; Prilusky et al., 1042 2005).

1043 C, D. Representative images (C) and quantification (D) of Tom70-GFP in young and old cells 1044 from wild type and mutant strains. Mean and s.e.m. from 57, 154, 50 old cells were normalized 1045 to young cells for each strain. Both young and old cells were from YPD medium.

1046 E, F. Representative images (E) and quantification (F) of Dnm1-GFP in young and old cells.

1047 Mean and s.e.m. from 89 young and 53 old cells were quantified. Both young and old cells were 1048 from YPD medium.

1049 G. Model of Tom70's roles. Tom70 sits at the crossroad of cytosolic proteostasis and 1050 mitochondrial biogenesis by regulating both the biogenesis and import of mitochondrial proteins. 1051 The reduction of Tom70 during aging is associated with age-dependent mitochondrial defects.

1052 Scale bar for all images: $5 \mu \mathrm{m}$. Mitochondrial proteins were visualized by endogenous C1053 terminal GFP tagging and expressed from their own promoters. Images are representative of at 1054 least two independent experiments. Data were analyzed with unpaired two-tailed t test: ***, $1055 \mathrm{p}<0.001 ; * *, \mathrm{p}<0.01 ; *, \mathrm{p}<0.05$; n.s., not significant. 
bioRxiv preprint doi: https://doi.org/10.1101/2021.11.23.469672; this version posted November 23, 2021. The copyright holder for this preprint (which was not certified by peer review) is the author/funder, who has granted bioRxiv a license to display the preprint in perpetuity. It is made available under aCC-BY 4.0 International license.

Figure 1-figure supplement 1

A

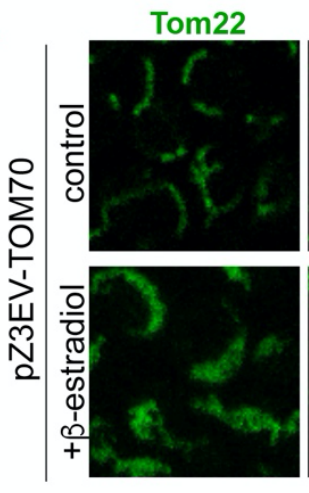

Cox5a

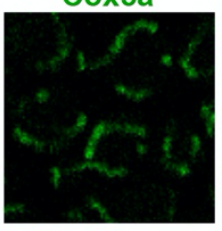

Ccp1

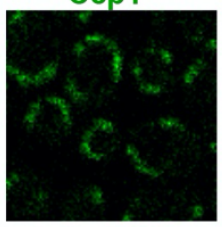

Leu9
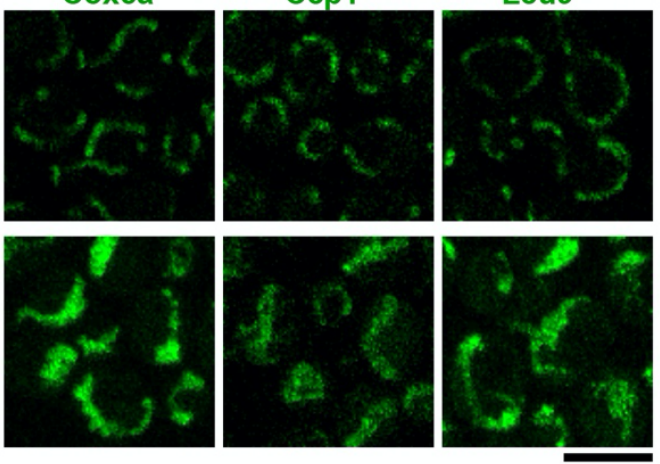

B

Tom20tm

-TEVprotease

C MEF2- UAS-

GAL4 TOM70

w1118 UAS-
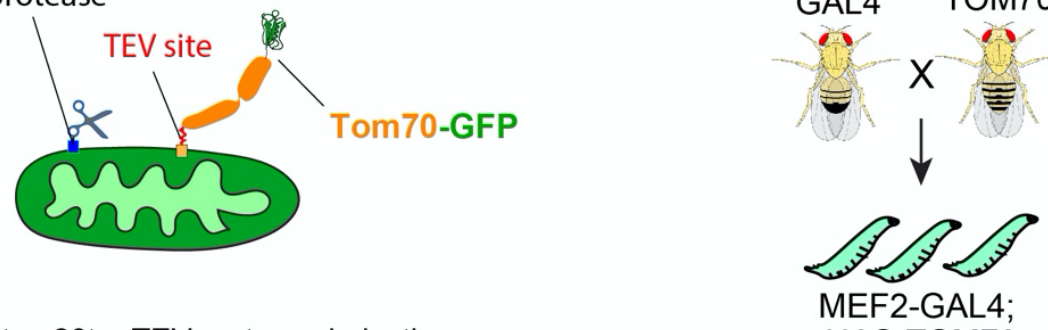

tom20tm-TEV protease induction

UAS-TOM70

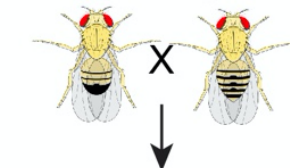

VS

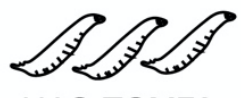

UAS-TOM70

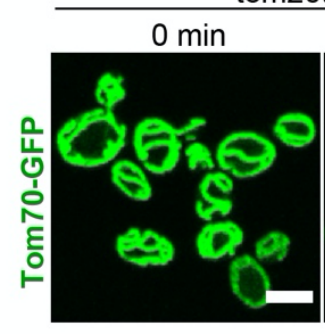
$20 \mathrm{~min}$ $40 \mathrm{~min}$

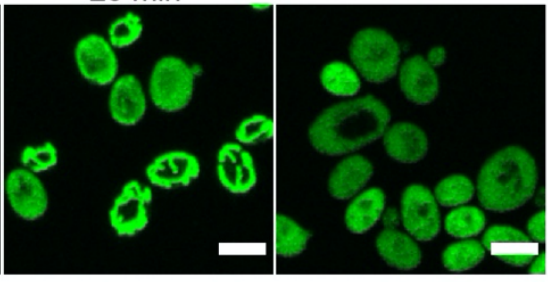

D
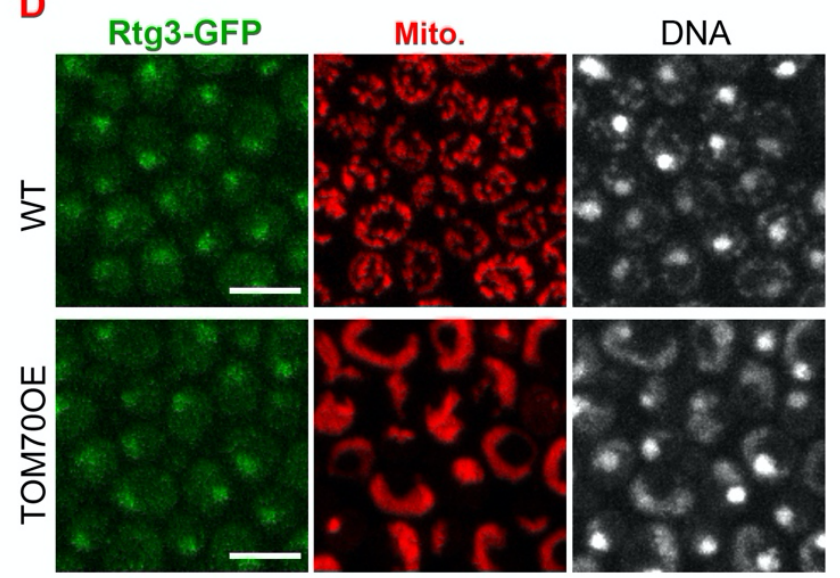

E

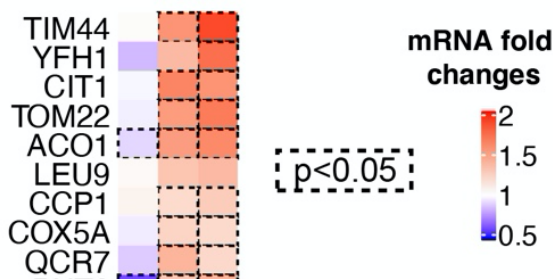


Figure 1-figure supplement 1: Additional data demonstrating that Tom70 regulates the transcription of mitochondrial proteins.

1072 A. Representative images for proteins localized to different sub-compartments of mitochondria

1073 in cells overexpressing Tom70 from pZ3EV promoter. Mitochondrial proteins were visualized

1074 by endogenous C-terminal GFP tagging and expressed from their native promoters. Cells were

1075 cultured in $2 \%$ raffinose and switched to $2 \%$ glucose and added $10 \mathrm{nM} \beta$-estradiol to induce the

1076 Tom70 expression for $5 \mathrm{hrs}$.

1077 B. Cleavage of Tom70-GFP that contains a TEV site between transmembrane and cytosolic

1078 domain by TEV protease. TEV protease was fused to Tom20 transmembrane domain (Tom20tm)

1079 and expressed from a Gal promoter. Expression of TEV protease was induced and the

1080 localization of Tom70-GFP was monitored at different timepoints.

1081 C. The genetic cross to generate $3^{\text {rd }}$ instar larvae from control (UAS-TOM70) and TOM70 OE

1082 (MEF2-GAL4; UAS-TOM70).

1083 D. Representative images of Rtg3-GFP in control and TOM70 OE cells. Rtg3-GFP, endogenous

1084 C-terminal GFP tagging of Rtg3. DNA was stained with Hoechst dye. All different yeast strains,

1085 including wild type control, were cultured in the same medium.

1086 E. qPCR quantification of different mitochondrial mRNA in wild type and rtg3 $\Delta$ cells that

1087 overexpress TOM70. The mRNA abundance was normalized to wild type control and the fold

1088 changes from three biological replicates are shown. Dash line boxes indicate the ones with

$1089 \mathrm{p}<0.05$ from unpaired two-tailed t test.

1090 Scale bar for all images: $5 \mu \mathrm{m}$. Mitochondrial proteins were visualized by endogenous C-

1091 terminal GFP tagging and expressed from their own promoters. Images are representative of at

1092 least two independent experiments.

1093

1094

1095

1096

1097

1098

1099

1100 
Figure 4-figure supplement 1
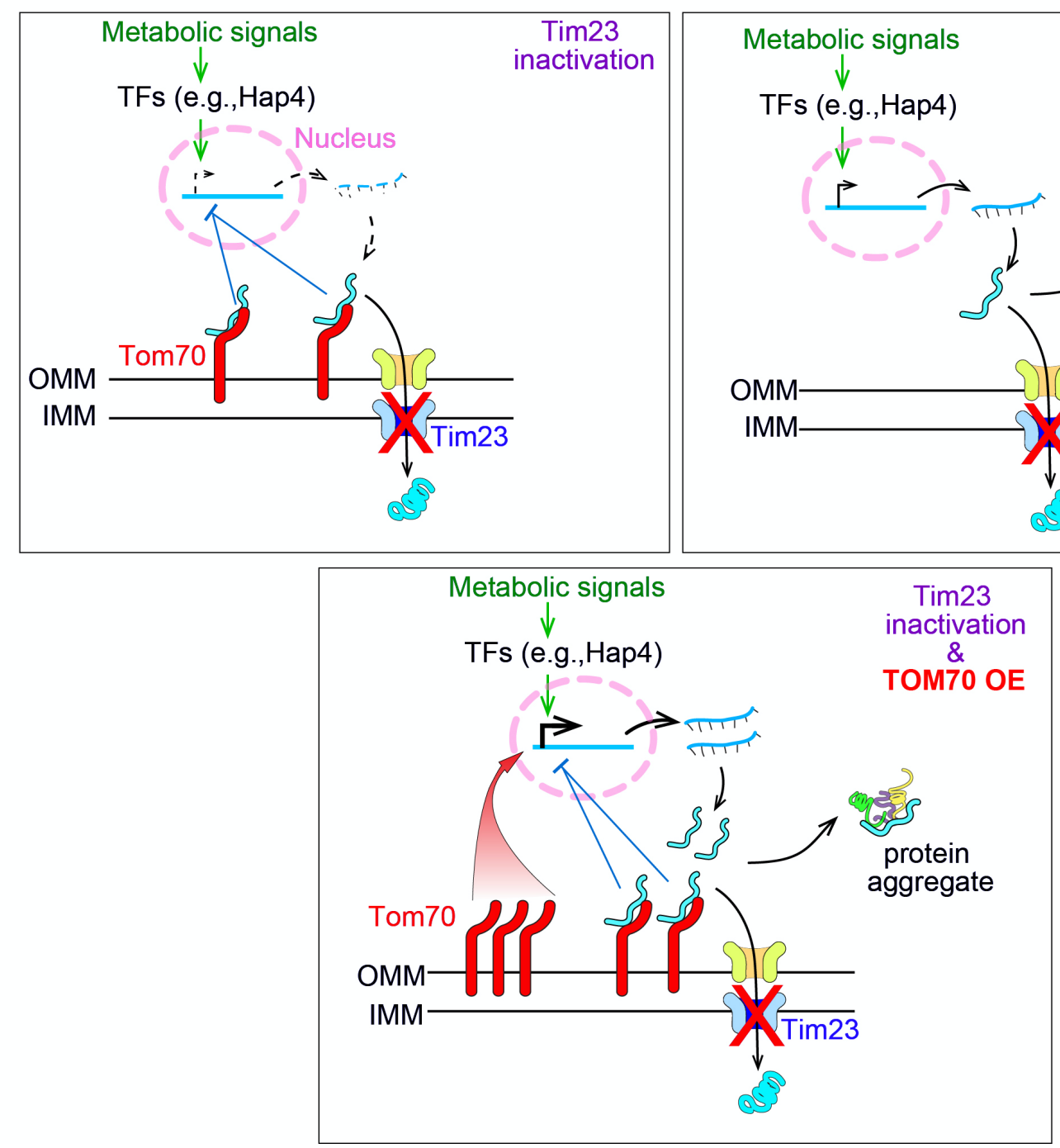

1102 Figure 4-figure supplement 1: Model of Tom70's role in the cellular response to the mitochondrial import defect based on the literature and our data.

1104 The inactivation of Tim23 blocks the mitochondrial import of nascent mitochondrial proteins,

1105 which occupy Tom70 and signal the nucleus to reduce the biogenesis of mitochondrial proteins.

1106 This allows the tim $23^{t s}$ cells to re-balance the biogenesis and import of mitochondrial proteins,

1107 thereby avoiding their cytosolic accumulation and aggregation. Tom70 is required for this

1108 mitochondria-to-nucleus signaling as knockout of Tom70 in tim $23^{\text {ts }}$ cells compromises this re-

1109 balancing effort and causes cytosolic protein aggregation. The fact that overexpression of

1110 TOM70, which activates the transcriptional activity of mitochondrial proteins, compromises the

1111 re-balancing effort of $\operatorname{tim} 23^{\text {ts }}$ cells is consistent with previous reports that transcriptional 
1112 repression of mitochondrial proteins is a key feature of the cellular response towards

1113 mitochondrial import defects (Lin et al., 2002; Ruetenik and Barrientos, 2015; Wuttke et al.,

1114 2012).

Figure 5-figure supplement 1
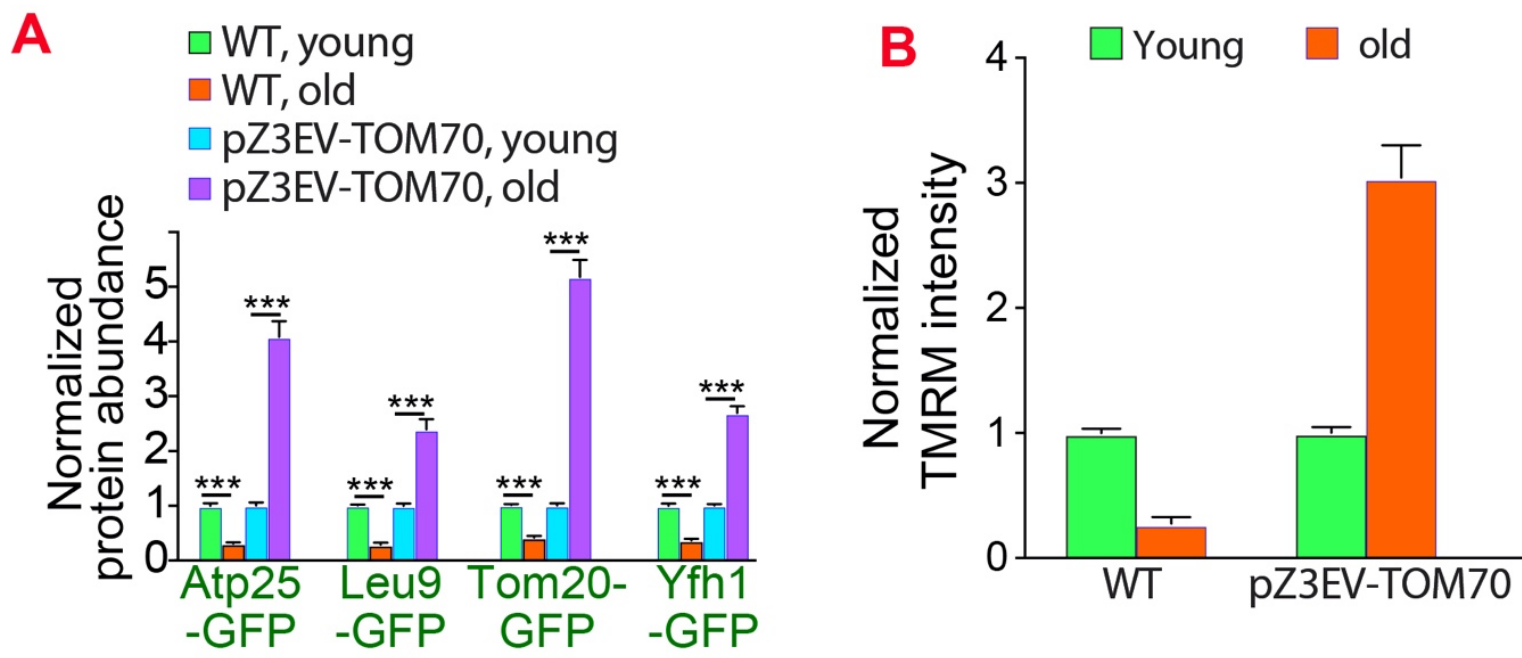

Figure 5-figure supplement 1: Overexpressing Tom70 from a different promoter can

1123 rescue age-associated cellular defects.

1124 A, B. Quantification of mitochondrial proteins abundance (A) and membrane potential (B) in

1125 different cells. The age-associated loss of mitochondrial membrane potential and protein

1126 expression can be suppressed by overexpressing TOM70 from pZ3EV promoter in the glucose

1127 medium. Both wild type (WT) and pZ3EV-TOM70 strains were cultured in YPD supplemented

1128 with $100 \mathrm{nM} \beta$-estradiol before and after biotinylating mother cell wall and culturing for 2 days

1129 to age the mother cells. Bar graphs are the mean and s.e.m. that normalized to young cells for

1130 each genotype. Data were analyzed with unpaired two-tailed test: ***, p<0.001; **, p<0.01; *, $1131 \mathrm{p}<0.05$; n.s., not significant. Sample sizes are given in Table S4. 
bioRxiv preprint doi: https://doi.org/10.1101/2021.11.23.469672; this version posted November 23, 2021. The copyright holder for this preprint (which was not certified by peer review) is the author/funder, who has granted bioRxiv a license to display the preprint in perpetuity. It is made available under aCC-BY 4.0 International license.

Figure 6-figure supplement 1

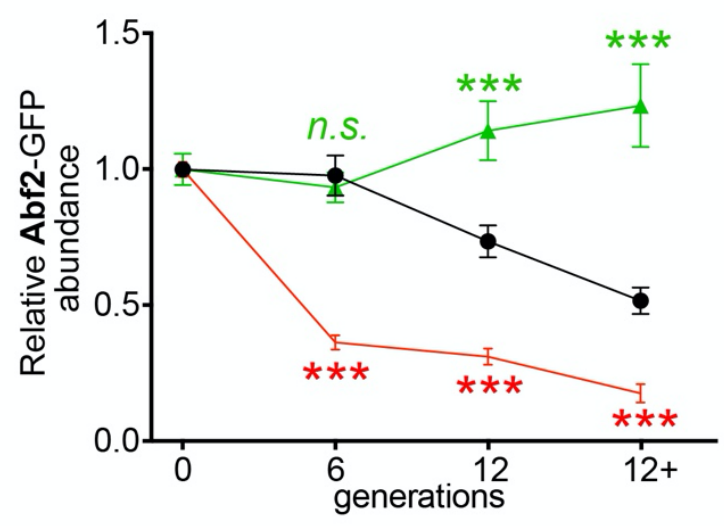

$-\Delta t o m 70 \quad-\Delta t o m 70 ; \mathrm{CR}$
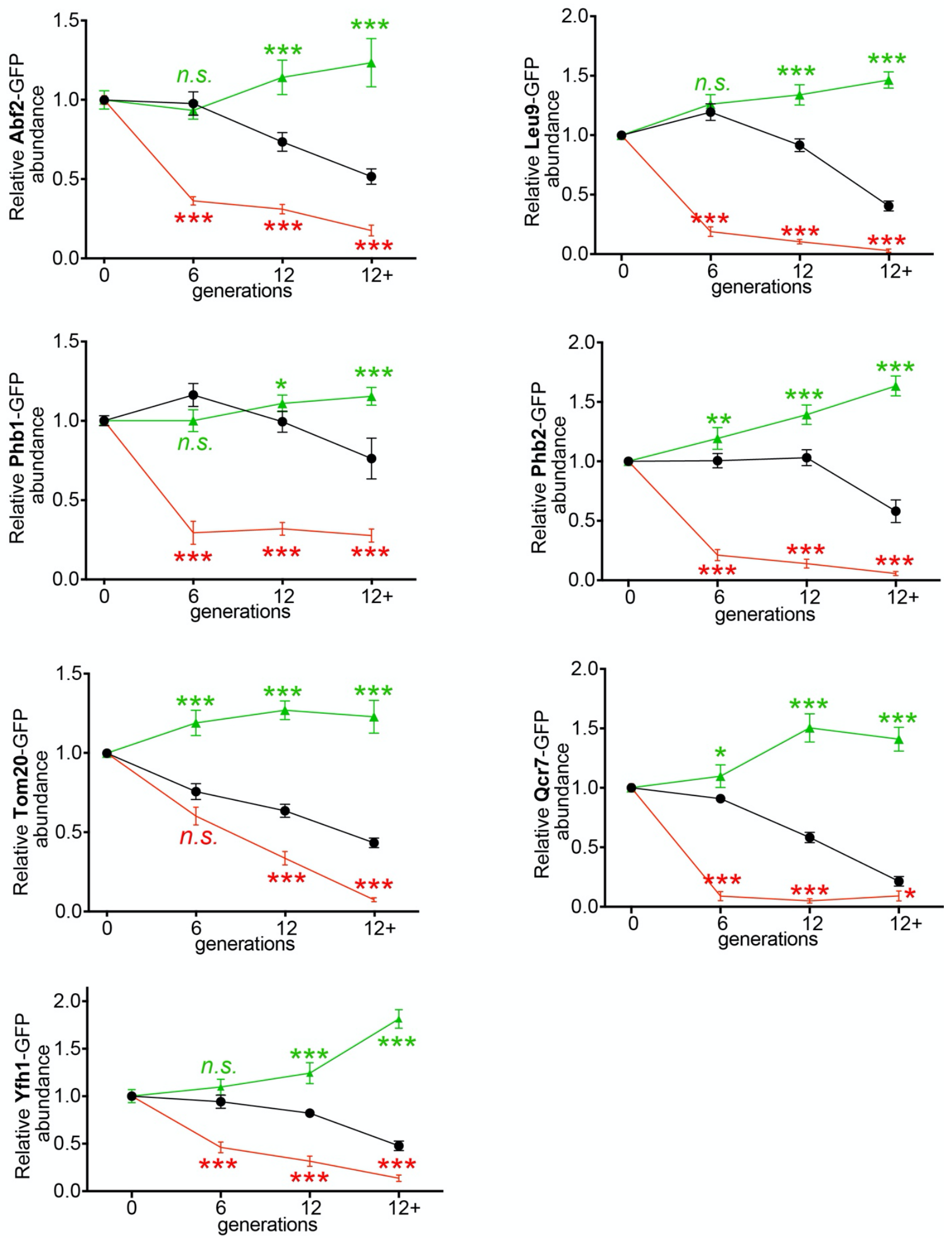


\section{Figure 6-figure supplement 1: Additional data for the accelerated loss of mitochondrial}

\section{5 proteins in $\Delta t o m 70$ cells during aging.}

1136 Quantifications of different mitochondrial proteins in young and aged cells of different strains.

1137 Cells with advanced reproductive age were purified from YPD culture and stained with

1138 calcofluor white to visualize the bud scars. Both young and old cells were quantified from the

1139 purified cells that went through the same experimental procedures and purification (same for

1140 other results). All strains cultured in YPD medium with 2\% glucose except for CR, which has

$11410.05 \%$ glucose. The GFP signal quantified from the old cells was normalized to corresponding

1142 young cells of the same strain. Old cells were grouped as young ( 0 bud scar), age 2-6, age 7-11,

1143 and age $12+$. More than 20 cells quantified for each group. 
Figure 7-figure supplement 1

A

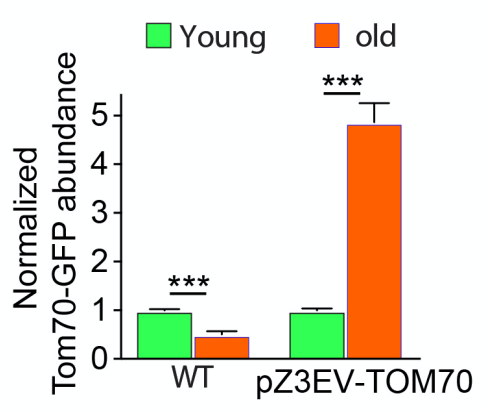

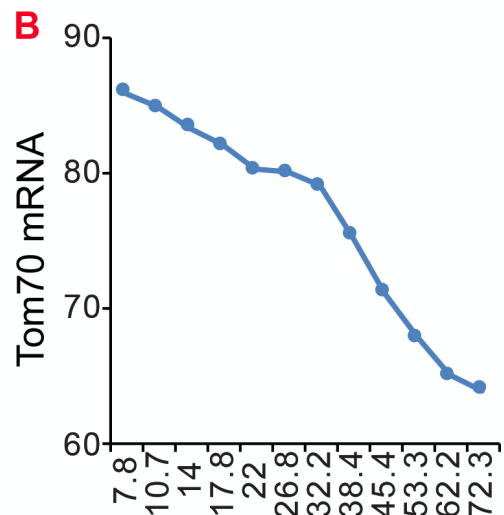

aging (hours)
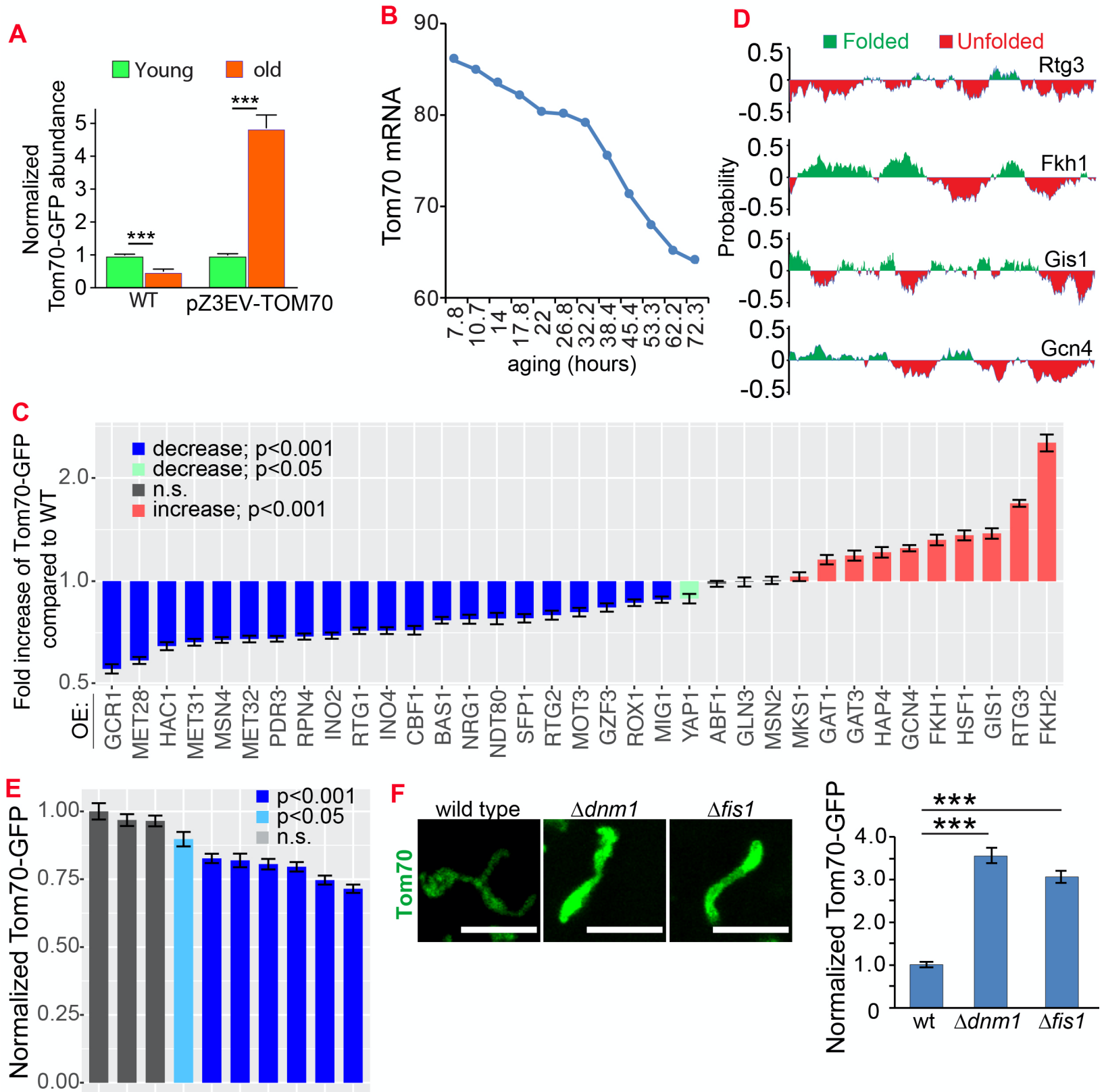

F
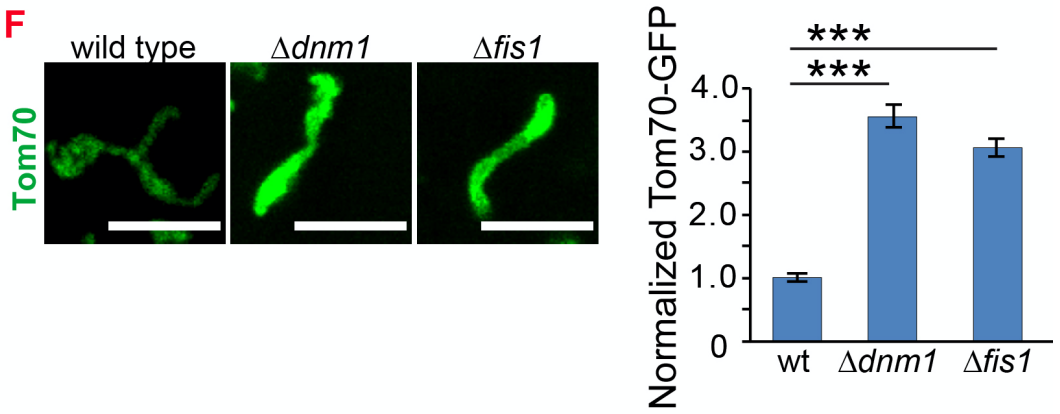

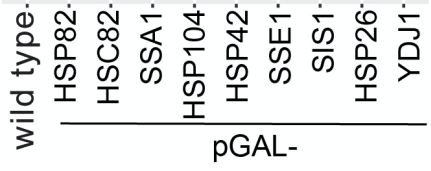

1164 Figure 7-figure supplement 1: Additional data for the mechanisms that control the expression of Tom70 in young and aged cells.

1166 A. Expressing TOM70-GFP from pZ3EV can prevent the age-associated reduction of Tom70

1167 protein. Both young and old cells were from YPD medium with $10 \mathrm{nM} \beta$-estradiol. 
1168 B. Tom 70 mRNA quantified from cells with different replicative ages. RNAseq data from

1169 Janssens et al., 2015 was plotted.

1170 C. Quantification of Tom70-GFP in young cells cultured in YEP-galactose medium to

1171 overexpress different TFs from the pGAL promoter. Shown are mean and s.e.m. normalized to

1172 wild type cells.

1173 D. Additional predicted folding index of different proteins.

1174 E. Tom70-GFP level in young cells cultured in YEP-galactose medium to overexpress different

1175 cytosolic chaperones from the pGAL promoter. Shown are normalized mean and s.e.m.

1176 F. Representative images and quantification of Tom70-GFP in young cells from wild type and

1177 mutant strains cultured in YPD medium. Mean and s.e.m. from 53, 50, 50 cells were quantified.

1178 WT, wild type.

1179 Scale bar for all images: $5 \mu \mathrm{m}$. Sample sizes for (A, C, E) are given in Table S4. Images are

1180 representative of at least two independent experiments. Data were analyzed with unpaired two-

1181 tailed t test: $* * *, \mathrm{p}<0.001 ; * *, \mathrm{p}<0.01 ; *, \mathrm{p}<0.05$; n.s., not significant.

\section{Supplemental tables}

Table_S1_Experimental Models- Organisms and Strains.xlsx.

Table_S2_OligoDNA.xlsx.

Table_S3_recombineDNA.

Table_S4_Source_Data_for_sample_sizes_related_to_several_figures.xlsx.

Table_S5_OligoDNA for fly genes tested. 